\title{
ARTICLE \\ Kinetics of the Oxidation of Hydrogen Sulfide by Atmospheric Oxygen in an Aqueous Medium
}

\section{Deepak Singh Rathore Vimlesh Kumar Meena Chandra Pal Singh Chandel Krishna Swarup Gupta*}

Atmospheric Chemistry Laboratory, Department of Chemistry, University of Rajasthan, Jaipur, 302004, India

\section{ARTICLE INFO}

Article history

Received: 15 July 2021

Accepted: 12 August 2021

Published Online: 16 August 2021

Keywords:

Hydrogen sulfide

Oxygen

Oxidation

Kinetics

Effect of organics

\begin{abstract}
Hydrogen sulfide is an important acid rain precursor and this led us to investigate the kinetics of its oxidation in aqueous phase by atmospheric oxygen. The kinetics was followed by measuring the depletion of oxygen in a reactor. The reaction was studied under pseudo order conditions with $\left[\mathrm{H}_{2} \mathrm{~S}\right]$ in excess. The kinetics followed the rate law:

$-\mathrm{d}\left[\mathrm{O}_{2}\right] / \mathrm{dt}=k[\mathrm{~S}]\left[\mathrm{O}_{2}\right]_{\mathrm{t}}$

Where $[\mathrm{S}]$ represents the total concentration of hydrogen sulfide, $\left[\mathrm{O}_{2}\right]_{\mathrm{t}}$ is the concentration of oxygen at time $t$ and $k$ is the second order rate constant.

The equilibria $(\mathrm{B}-\mathrm{C})$ govern the dissolution of $\mathrm{H}_{2} \mathrm{~S}$; the sulfide ion in water forms different species:

$\mathrm{H}_{2} \mathrm{~S} \stackrel{\mathrm{K}_{1}}{\rightleftharpoons} \mathrm{HS}^{-}+\mathrm{H}^{-}$

$\mathrm{HS}^{-} \stackrel{\mathrm{K}_{2}}{\rightleftharpoons} \mathrm{S}^{2-}+\mathrm{H}^{-}$

Where $\mathrm{K}_{1}$ and $\mathrm{K}_{2}$ are first and second dissociation constants of $\mathrm{H}_{2} \mathrm{~S}$.

Although, $\mathrm{H}_{2} \mathrm{~S}$ is present as undissociated $\mathrm{H}_{2} \mathrm{~S}, \mathrm{HS}^{-}$and $\mathrm{S}^{2-}$ ions, nature of $\left[\mathrm{H}^{+}\right]$ dependence of reaction rate required only $\mathrm{HS}^{-}$to be reactive and dominant. The rate law (A) on including $\left[\mathrm{H}^{+}\right]$dependence became Equation (D).

$-\mathrm{d}\left[\mathrm{O}_{2}\right] / \mathrm{dt}=k_{1} \mathrm{~K}_{1}\left[\mathrm{H}^{+}\right][\mathrm{S}]\left[\mathrm{O}_{2}\right]_{\mathrm{t}} /\left(\left[\mathrm{H}^{+}\right]^{2}+\mathrm{K}_{1}\left[\mathrm{H}^{+}\right]+\mathrm{K}_{1} \mathrm{~K}_{2}\right)$

Our results indicate anthropogenic VOCs such as acetanilide, benzene, ethanol, aniline, toluene, benzamide, $o$-xylene, $m$-xylene, $p$-xylene and anisole to have no significant effect on the reaction rate and any observed small effect is within the uncertainty of the rate measurements.

\section{Introduction}

There are several reasons for undertaking studies on the aqueous phase atmospheric oxidation of hydrogen sulfide, $\mathrm{H}_{2} \mathrm{~S}$, by oxygen. Firstly, along with sulfur dioxide, $\mathrm{SO}_{2}$, and dimethyl sulfide, $\mathrm{CH}_{3} \mathrm{SCH}_{3}$, it is among the major sulfur - based trace atmospheric gases, which are all acid rain precursors. $\mathrm{H}_{2} \mathrm{~S}$ is emitted by both natural and anthropogenic sources. The natural sources of $\mathrm{H}_{2} \mathrm{~S}$ include the anaerobic degradation of organic matter ${ }^{[1]}$, sulfur springs, swamps, volcanoes, natural gas, some well waters, geothermal active areas, etc. The anaerobic biogenic reactions in coastal wetlands, soils and plants, volcanoes, and biomass burning ${ }^{[2]}$, produce reduced sulfur compounds like $\mathrm{H}_{2} \mathrm{~S}, \mathrm{COS}$, DMS, etc. The sulfide minerals, which are the most important group of ore

*Corresponding Author:

Krishna Swarup Gupta,

Atmospheric Chemistry Laboratory, Department of Chemistry, University of Rajasthan, Jaipur, 302004, India;

Email: guptaks200@gmail.com 
minerals, are the other natural source of sulfides. Several industrial processes such as coke production, viscose rayon production, wood pulp production, sulfur extraction processes, oil- refining, tanning, oil and gas drilling, production and refining, liquid manure are the man made sources of $\mathrm{H}_{2} \mathrm{~S}$. The stagnant bodies of water and sewers also release $\mathrm{H}_{2} \mathrm{~S}$.

Secondly, bacterial breakdown of organic materials in human and animal wastes, sewage, stagnant wastewater and sewers is responsible of toxic $\mathrm{H}_{2} \mathrm{~S}$ and its foul smell. It affects the human health adversely and causes health hazards.

Thirdly, whereas the atmospheric chemistry of aqueous $\mathrm{SO}_{2}$ has been the subject of extensive studies ${ }^{[3-5]}$, the same is not true of $\mathrm{H}_{2} \mathrm{~S}$. The oxidations of $\mathrm{SO}_{2}$ directly and that of $\mathrm{H}_{2} \mathrm{~S}$ via $\mathrm{SO}_{2}$ in to sulfate are responsible for acidification of atmospheric waters and formation of sulfate aerosols. Obviously, for understanding the aqueous phase atmospheric oxidation of $\mathrm{H}_{2} \mathrm{~S}$ by oxygen, there are some aspects, which from atmospheric chemistry point of view need serious reinvestigation under ambient experimental conditions of $\mathrm{pH},\left[\mathrm{O}_{2}\right]$, anthropogenic VOCs, etc, which mimic atmospheric waters.

Fourthly, the atmospheric chemistry of hydrogen sulfide plays an important role in sulfur cycle. Along with other reduced sulfur compounds, it is oxidized to $\mathrm{SO}_{2}$, and thereafter largely to sulfate. Organization for Economic Co-operation and Development(OECD) ${ }^{[6]}$ estimates that the origin of about half of the global $\mathrm{SO}_{2}$ is the oxidation of $\mathrm{H}_{2} \mathrm{~S}$. Lifetime of $\mathrm{H}_{2} \mathrm{~S}$ is $\sim 4.4$ days. Thus, $\mathrm{H}_{2} \mathrm{~S}$ is one of the main precursors of $\mathrm{SO}_{2}$ and sulfate in atmosphere ${ }^{[7]}$.

Finally, volatile organic compounds intervene and influence the atmospheric chemistry of acid rain ${ }^{[8]}$, photochemical smog ${ }^{[9]}$, etc. Even then, there appears to be only few cursory brief reports on the role of some organic compounds on the inhibition of the autoxidation of $\mathrm{H}_{2} \mathrm{~S}^{[10,11]}$. Similarly, on the role of ammonia no report has come to our notice.

Generally, very low ambient concentrations of hydrogen sulfide are found in the atmosphere. While the ambient $\mathrm{H}_{2} \mathrm{~S}$ concentrations range from $0.11 \mathrm{ppb}$ to 0.33 $\mathrm{ppb}$ in rural areas, the values are generally less than 1 ppb in urban areas ${ }^{[12]}$. In some cases, relatively very high concentrations, exceeding $90 \mathrm{ppb}$, have been reported near natural sources and $\mathrm{H}_{2} \mathrm{~S}$ releasing industries ${ }^{[13]}$.

Oxygen readily oxidizes hydrogen sulfide. A decrease in $\mathrm{pH}$ decreases the rate of hydrogen sulfide oxidation ${ }^{[14]}$, which may have implications for sulfide ore mines with acid mine drainage; in the latter case, the acid would be sulfuric acid, but hydrogen sulfide gas may still be present. The oxidation of $\mathrm{H}_{2} \mathrm{~S}$ by $\mathrm{O}_{2}$, i.e., autoxidation of $\mathrm{H}_{2} \mathrm{~S}$, takes place in all forms of atmospheric waters resulting in a variety of oxidation products such as sulfur, $\mathrm{S}^{\mathrm{o}}$, sulfite ion, $\mathrm{SO}_{3}{ }^{2-}$, thiosulfate ion, $\mathrm{S}_{2} \mathrm{O}_{3}{ }^{2-}$, and sulfate ion, $\mathrm{SO}_{4}{ }^{2-}$, etc. ${ }^{[15-20]}$. This reaction is of great significance in seawater ${ }^{[21-29]}$ and wastewater ${ }^{[30,31]}$

The oxidation of $\mathrm{H}_{2} \mathrm{~S}$ by other oxidants such as chromate(VI) ${ }^{[32]}$, ferrate(VI) ${ }^{[33]}$, hydrogen peroxide ${ }^{[34]}$ and peroxomonosulfate ${ }^{[35]}$ has also been studied. Tomar and Abdullah ${ }^{[36]}$ found the oxidation of $\mathrm{H}_{2} \mathrm{~S}$ in wastewater to be slow.

The results of reported $\mathrm{H}_{2} \mathrm{~S}$ autoxidation kinetics studies differ widely although in general kinetics order in each of $\mathrm{H}_{2} \mathrm{~S}$ and $\mathrm{O}_{2}$ is reported to be one ${ }^{[21,37-39]}$ although some studies report higher, lower or complex orders ${ }^{[16]}$. Some studies have been carried out with highly pure solutions and reagents ${ }^{[17]}$. Luther et al. ${ }^{[37]}$ reported that in the absence of trace metals the reaction is very slow. There are conflicting reports about induction period and colloidal sulfur formation. Whereas, Chen and Morris ${ }^{[16]}$ in their buffered study of uncatalyzed reaction in the $\mathrm{pH}$ range 6-13 observed an induction period of up to one hour, under similar reaction conditions O'Brien and Birkner ${ }^{[18]}$ $(\mathrm{pH}=4-10)$ found no induction period. Whereas, Chen and Morris ${ }^{[16]}$, Millero ${ }^{[21]}$ reported the polysulfide formation, Avrahami and Golding ${ }^{[17]}$, O'Brien and Birkner ${ }^{[18]}$ did not report any such phenomenon. As regards the products, sulfate was reported as a final product ${ }^{[17,18]}$.

This led us to look afresh into the atmospheric chemistry of $\mathrm{H}_{2} \mathrm{~S}$ oxidation and to examine the effect of several parameters such as reactant concentrations, $\mathrm{pH}$ and importantly the effect of some anthropogenic volatile organic compounds(VOCs) particularly those which are reported to strongly inhibit the oxidation of aqueous $\mathrm{SO}_{2}$ by oxygen in laboratory water. The oxidation kinetics of aqueous $\mathrm{SO}_{2}$ and effect of VOCs has been studied by several groups ${ }^{[3,8,40-57]}$.

\section{Experimental}

The experimental procedure for studying the kinetics of aqueous hydrogen sulfide oxidation by oxygen was same as described previously ${ }^{[58]}$. Briefly it is described here. Reactions were conducted in air-tight sealed three necked glass reactor of $250 \mathrm{ml}$ capacity which was immersed in a thermostated water bath to maintain the desired temperature $( \pm 0.1)$. The dissolved oxygen probe was fitted in one neck and the oxygen from a cylinder was passed through the other neck. The third neck was used for adding standard sodium sulfide solution. The procedure for studying kinetics was as follows. At first the oxygen was passed in the reactor till the desired concentration of $\mathrm{O}_{2}$ was achieved. Immediately, afterwards, the 
standardized $\mathrm{Na}_{2} \mathrm{~S}$ solution was added and the reactor sealed. The kinetics was followed by recording decrease in $\mathrm{O}_{2}$ concentration by DO meter (Durox Oxi-3205).

In lieu of hydrogen sulfide, sodium sulfide was used, which generated sulfide species in aqueous solution through hydrolysis of sulfide ion, $\mathrm{S}^{2-}$.

$\mathrm{S}^{2-}+\mathrm{H}_{2} \mathrm{O} \rightleftharpoons \mathrm{HS}^{-}+\mathrm{OH}^{-}$

$\mathrm{HS}^{-}+\mathrm{H}_{2} \mathrm{O} \rightleftharpoons \mathrm{H}_{2} \mathrm{~S}+\mathrm{OH}^{-}$

The solution of $\mathrm{Na}_{2} \mathrm{~S}$ was prepared in double distilled water daily afresh.

The replicate rate determinations were generally reproducible within $\pm 20 \%$. As discussed later the high uncertainty results from the catalytic activity of trace metal ion impurities ${ }^{[59]}$. All calculations were performed on MS Excel 2007. The values of rate coefficients together with standard deviations and correlation coefficients have been presented.

\subsection{Product Analysis}

The kinetics of this reaction has been studied under deficit $\left[\mathrm{O}_{2}\right]$ at a ratio $\left[\mathrm{O}_{2}\right] /[\mathrm{S}] \approx 0.012-0.12$, where $\left[\mathrm{O}_{2}\right]$ and $[\mathrm{S}]$ represent initial $\mathrm{O}_{2}$ and $\mathrm{H}_{2} \mathrm{~S}$ concentrations, respectively. This $\left[\mathrm{O}_{2}\right] /[\mathrm{S}]$ ratio was optimum for studying kinetics study although it is much different than the atmospheric ratio. The products were identified by running the experiments at $[\mathrm{S}]=2 \times 10^{-3} \mathrm{~mol} \mathrm{~L}^{-1}$ and $\left[\mathrm{O}_{2}\right]$ $=2 \times 10^{-4} \mathrm{~mol} \mathrm{~L}^{-1}$ at $\mathrm{pH} \approx 10.6$ and $\mathrm{T}=30^{\circ} \mathrm{C}$. The reaction was allowed to reach completion. No change in color of the reaction mixture during the progress as well as at the end of the reaction was noticed. There was no appearance of turbidity showing that no formation of colloidal sulfur/ poly sulfides took place ${ }^{[11]}$ and the mixture remained transparent. Thus, our experimental conditions do not appear to favor colloidal sulfur formation. Although the formation of polysulfides species in solutions of $\mathrm{pH}$ more than 8 has been noted ${ }^{[16]}$, stoichiometry of sulfide-oxygen reaction depends on $\mathrm{pH}$, concentration of reactants, and the existence of extraneous substances or impurities. The sulfite ion, which is an important intermediate, could not be detected. Probably, even if it is formed, it is oxidized to sulfate ${ }^{[16]}$. So, sulfate was found to be the only product, which could be detected.

Since $\left[\mathrm{O}_{2}\right]$ was low $\left(\approx 10^{-4} \mathrm{~mol} \mathrm{~L}^{-1}\right)$ any oxidation product formed would also be low. To collect a measurable amount of the products, these experiments were repeated ten times. All final reaction solutions were mixed. And the resultant solution was acidified and heated to expel sulfide as $\mathrm{H}_{2} \mathrm{~S}$. Sulfate was detected as a product by precipitating it as insoluble $\mathrm{BaSO}_{4}$ and following the usual procedure. Like the present work, there are many other studies which also indicate the formation of sulfate as a major product in alkaline media. Zhang and Millero ${ }^{[60]}$ reported that the final product from the oxidation of sulfide is sulfate.

Although many intermediates such as sulfite, thiosulfate, sulfur (0), polysulfides etc. have been proposed for this reaction, sulfite has been considered as a initial product, which is oxidized ultimately to sulfate ${ }^{[6]]}$.

\section{Results}

\subsection{Preliminary Investigations}

Kinetics was studied under pseudo first order conditions and the oxygen was in deficit. Concentration of sulfide was in excess at least ten times over oxygen concentration. Hereafter, [S] represents the total $\mathrm{H}_{2} \mathrm{~S}$ concentration including its all species. The rate of oxidation was measured by following the disappearance of $\left[\mathrm{O}_{2}\right]$. In most cases, the reaction obeyed a first order kinetics in $\left[\mathrm{O}_{2}\right]$ (Figure 1). Values of pseudo first order rate constants, $k_{\mathrm{obs}}$, were obtained from $\log \left[\mathrm{O}_{2}\right]_{\mathrm{t}}$ versus time, $t$, plots defined by Equation (3).

$-\mathrm{d}\left[\mathrm{O}_{2}\right] / \mathrm{dt}=k_{\mathrm{obs}}\left[\mathrm{O}_{2}\right]_{\mathrm{t}}$

Where $\left[\mathrm{O}_{2}\right]_{\mathrm{t}}$ is concentration of oxygen at time, $\mathrm{t}$.

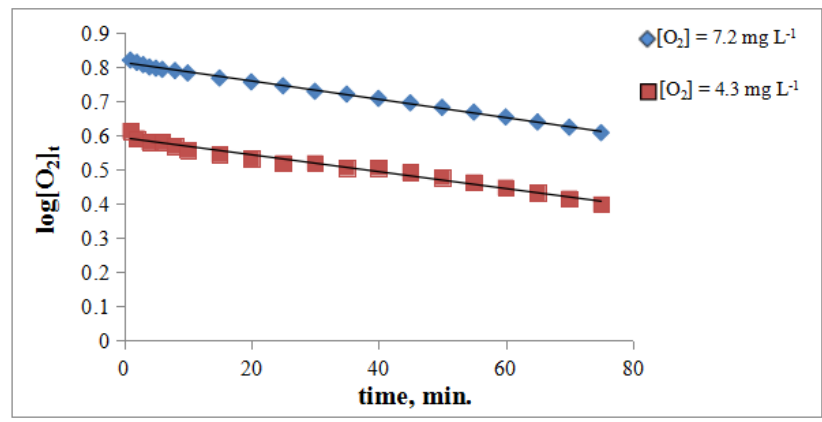

Figure 1. The plot of $\log \left[\mathrm{O}_{2}\right]_{\mathrm{t}}$ versus time at $\mathrm{pH}=10.82$ $10.86,[\mathrm{~S}]=2 \times 10^{-3} \mathrm{~mol} \mathrm{~L}^{-1}$ and $\mathrm{T}=30^{\circ} \mathrm{C}$

The dependence of reaction rate on $\mathrm{pH}$ is an important parameter due to the equilibria (1-2). The initial forays on $\mathrm{pH}$ dependence were made by carrying out the variation in $\mathrm{pH}$ in both the unbuffered and phosphate buffered media. In case of buffered study, in the kinetics run, the initial $\mathrm{pH}$ was fixed with the help of phosphate buffer by fixing the desired initial $\mathrm{pH}( \pm 0.02)$ by varying $\left[\mathrm{Na}_{2} \mathrm{HPO}_{4}\right]$ $\left[\mathrm{NaH}_{2} \mathrm{PO}_{4}\right]$ ratio. In case of unbuffered study, the desired initial $\mathrm{pH}( \pm 0.02)$ of the reaction mixture was fixed by addition of dilute perchloric acid. In this case, $\mathrm{pH}$ decreased with the progress of the reaction.

The results of $\mathrm{pH}$ dependence of rate at almost similar initial $\mathrm{pH}$ of reaction mixtures in both buffered and unbuffered media are compared in Table 1, which shows $k_{\text {obs }}$ values to be relatively high (130\%) in buffered 
media. This is probably due to introduction of trace metal impurities, when the buffer is used. Similar to S(IV) autoxidation studies ${ }^{[3]}$, several previous studies report the catalysis of $\mathrm{H}_{2} \mathrm{~S}$ oxidation by trace metal ions ${ }^{[59]}$. Recent studies from our laboratory report the presence of catalytic metal ions such as iron, manganese, cobalt, copper, etc in rainwater samples collected at Jaipur ${ }^{[8,61,62-64]}$. The concentrations of metal ions in rainwater are larger than those in distilled water ${ }^{[64]}$.

In general, the homogenous trace metal catalyzed reactions are inhibited by Ethylenediamine tetraacetate (EDTA). Indeed the latter is reported to inhibit the sulfide autoxidation ${ }^{[11]}$. The catalytic metal ions present as impurity in water and reagent samples are strongly complexed by EDTA and rendered inactive resulting in inhibition. In this study, the addition of EDTA led to a decrease in the rate of oxidation of $\mathrm{H}_{2} \mathrm{~S}$ (Figure 2) and at $\left[\right.$ EDTA] $\geq 1 \times 10^{-5} \mathrm{~mol} \mathrm{~L}^{-1}$ the reaction was completely inhibited (Figure 3). This clearly indicates that the impurity metal ions catalyze the rate of this reaction ${ }^{[63]}$. In view of this, to avoid the introduction of impurity ions, the entire study has been carried out in an unbuffered medium without using any buffer.

\subsection{Rate Law}

To arrive at the rate law, at first the effect of [S] was

Table 1. Values of $k_{\mathrm{obs}}$ in phosphate buffered and unbuffered media at $[\mathrm{S}]=2 \times 10^{-3} \mathrm{~mol} \mathrm{~L}^{-1}$ and $\mathrm{T}=30^{\circ} \mathrm{C}$

\begin{tabular}{cccccc}
\hline \multirow{2}{*}{ Experiment } & \multicolumn{2}{c}{ Unbuffered medium } & \multicolumn{2}{c}{ Buffered medium } & \multirow{2}{*}{$k_{\text {obs(Buffered) }} / k_{\text {obs(Unbuffered) }}$} \\
\cline { 2 - 4 } & Initial pH & $k_{\mathrm{obs}}, \mathrm{s}^{-1}$ & Initial pH & $k_{\mathrm{obs}}, \mathrm{s}^{-1}$ & 1.53 \\
2 & 8.85 & $1.14 \times 10^{-4}$ & 8.83 & $1.74 \times 10^{-4}$ & 3.25 \\
3 & 8.26 & $9.94 \times 10^{-5}$ & 8.27 & $3.23 \times 10^{-4}$ & 2.02 \\
4 & 8.04 & $7.64 \times 10^{-5}$ & 8.01 & $1.53 \times 10^{-4}$ & 2.31 \\
& 7.7 & $8.37 \times 10^{-5}$ & 7.71 & $1.93 \times 10^{-4}$ & Average $=2.3 \pm 0.5$ \\
\hline
\end{tabular}

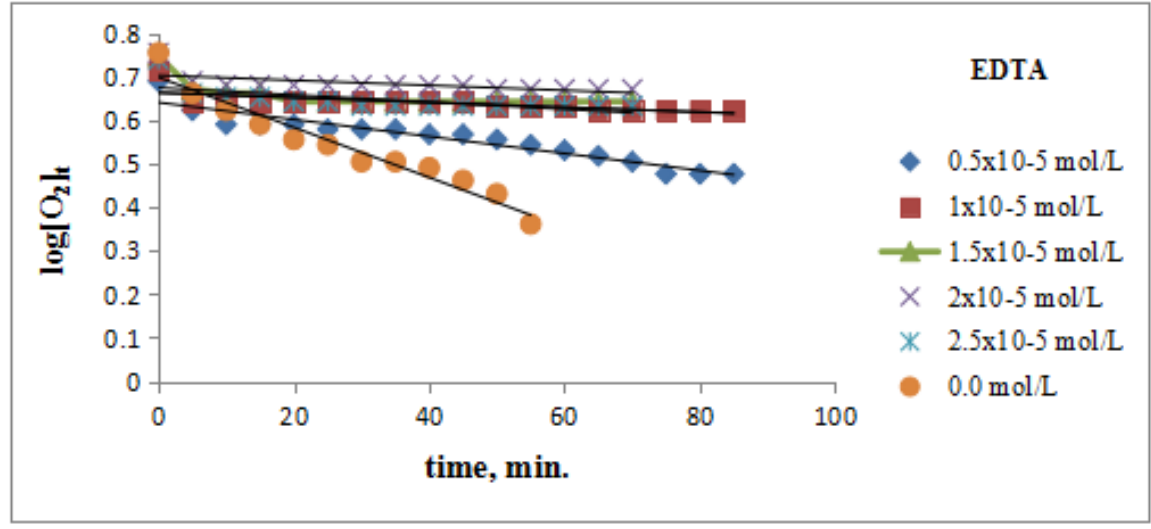

Figure 2. Effect of EDTA on the rate of $\mathrm{H}_{2} \mathrm{~S}$ autoxidation at $\mathrm{pH}=10.81-10.9$ at $[\mathrm{S}]=2 \times 10^{-3} \mathrm{~mol} \mathrm{~L}^{-1}$ and $\mathrm{T}=30^{\circ} \mathrm{C}$

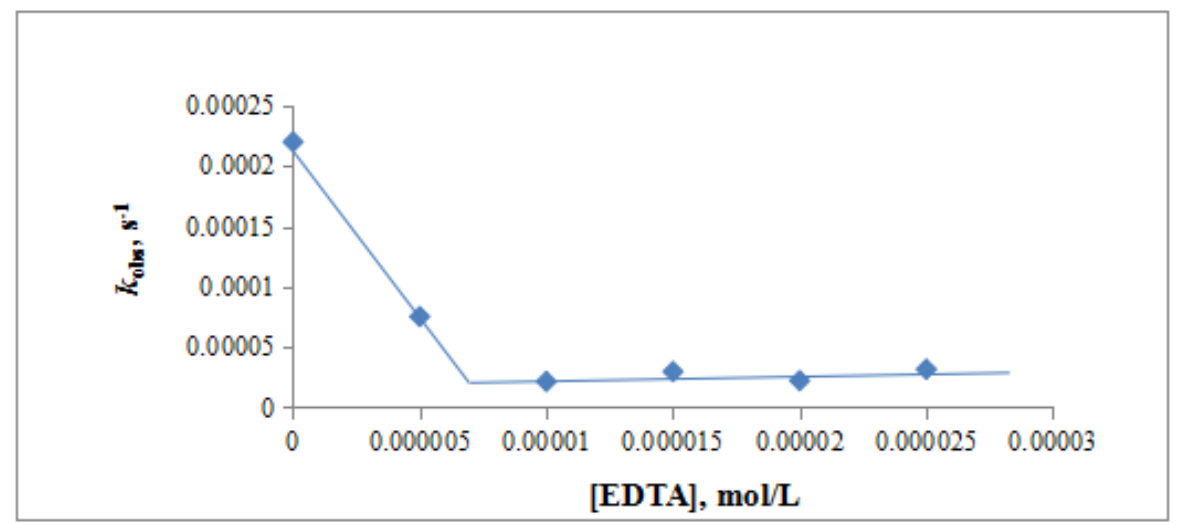

Figure 3. Effect of [EDTA] on $\mathrm{k}_{\mathrm{obs}}$ of $\mathrm{H}_{2} \mathrm{~S}$ autoxidation at $\mathrm{pH}=10.81-10.9$, $[\mathrm{S}]=2 \times 10^{-3} \mathrm{~mol} \mathrm{~L}^{-1}$ and $\mathrm{T}=30^{\circ} \mathrm{C}$ 
studied by varying $\mathrm{Na}_{2} \mathrm{~S}\left(2 \times 10^{-3}-8 \times 10^{-3} \mathrm{~mol} \mathrm{~L}^{-1}\right)$ in unbuffered medium. From eight $[\mathrm{S}]$-dependence rate studies (Table 2) at three temperatures, 25,30 and $35^{\circ} \mathrm{C}$, from $\log k_{\mathrm{obs}}$ versus $\log [\mathrm{S}]$ plots, the kinetics order was found to be $1.02 \pm 0.13$.

Table 2. The values of order in [S] determined from log $k_{\mathrm{obs}}$ versus $\log [\mathrm{S}]$ plots

\begin{tabular}{ccccccccc}
\hline Temp.,${ }^{\circ} \mathrm{C}$ & 25 & 25 & 30 & 30 & 30 & 30 & 30 & 35 \\
\hline Order in [S] & 1.25 & 0.58 & 0.98 & 1.28 & 0.78 & 1.54 & 0.76 & 0.96 \\
$\begin{array}{c}\text { Average order } \\
\text { in [S] }\end{array}$ & $1.02 \pm 0.13$ & & & & & & \\
\hline
\end{tabular}

Thus, [S] and $k_{\mathrm{obs}}$ are related by Equation (4).

$k_{\mathrm{obs}}=k[\mathrm{~S}]$

On combining Equations (3) and (4), we get Equation (5). $-\mathrm{d}\left[\mathrm{O}_{2}\right] / \mathrm{dt}=k[\mathrm{~S}]\left[\mathrm{O}_{2}\right]_{\mathrm{t}}$

In accordance with Equation 5, the plot of $k_{\mathrm{obs}}$ versus [S] is linear (Figure 4).

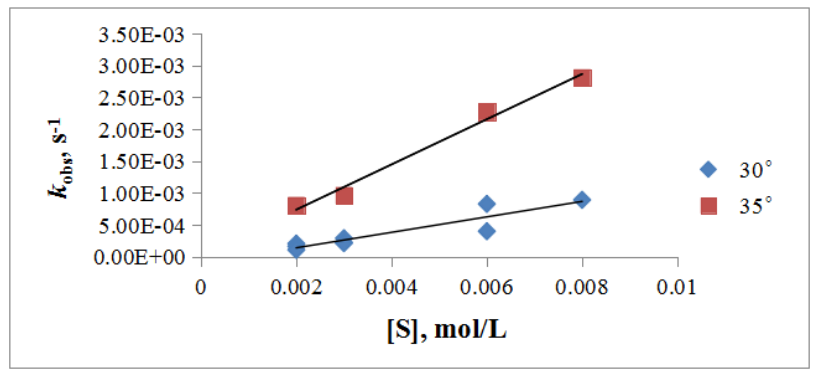

Figure 4. The plots of $k_{\mathrm{obs}}$ versus [S] at two different temperatures.

The values of $k_{\text {obs }}$ at different $[\mathrm{S}]$ and $\left[\mathrm{O}_{2}\right]$ are in Table 3 . Since the kinetics has been performed in unbuffered medium, attempts were made to keep the same initial $\mathrm{pH}$. However, despite our best efforts it was not possible to have exactly the same $\mathrm{pH}$ of the reaction mixtures, while varying $[\mathrm{S}]$ and $\left[\mathrm{O}_{2}\right]$. Aside from trace metal ions catalysis, this is also one of the reasons for greater than normal uncertainty and scatter in $k_{\text {obs }}$ values ${ }^{[59]}$.

\section{3 pH- Dependence}

To study the effect of $\mathrm{pH}$, the desired $\mathrm{pH}$ of water was adjusted with the help of dilute perchloric acid. In this process, the initial $\mathrm{pH}$ differed from the desired $\mathrm{pH}$ by about \pm 0.02 units. To take into account this $\mathrm{pH}$ difference, each experiment was repeated three times and the $k_{\text {obs }}$ values reported here are the average values of these replicate determinations. Since in each kinetics run, the $\mathrm{pH}$ changed with the progress of reaction, in the calculation of $\left[\mathrm{H}^{+}\right]$only the initial $\mathrm{pH}$ has been considered. $k_{\text {obs }}$ values at different $\mathrm{pH}$ (Table 4) are seen to increase
Table 3. The selected values of rate constants, $k_{\mathrm{obs}}$, at different $[\mathrm{S}]$ and $\left[\mathrm{O}_{2}\right]$

\begin{tabular}{|c|c|c|c|c|}
\hline $\begin{array}{c}\text { Temperature, } \\
{ }^{\circ} \mathrm{C}\end{array}$ & Initial $\mathrm{pH}$ & {$[\mathrm{S}], \mathrm{mol} \mathrm{L}^{-1}$} & {$\left[\mathrm{O}_{2}\right], \mathrm{mg} \mathrm{L}^{-1}$} & $k_{\mathrm{obs}}, \mathrm{s}^{-1}$ \\
\hline \multirow[t]{4}{*}{25} & 10.77 & $2 \times 10^{-3}$ & 6.22 & $0.54 \times 10^{-4}$ \\
\hline & 11.06 & $4 \times 10^{-3}$ & 6.31 & $1.39 \times 10^{-4}$ \\
\hline & 11.23 & $6 \times 10^{-3}$ & 5.98 & $1.92 \times 10^{-4}$ \\
\hline & 11.32 & $8 \times 10^{-3}$ & 6.25 & $3.32 \times 10^{-4}$ \\
\hline \multirow[t]{4}{*}{25} & 10.89 & $2 \times 10^{-3}$ & 5.5 & $3.66 \times 10^{-4}$ \\
\hline & 11.17 & $3 \times 10^{-3}$ & 5.5 & $5.14 \times 10^{-4}$ \\
\hline & 11.43 & $6 \times 10^{-3}$ & 5.5 & $7.41 \times 10^{-4}$ \\
\hline & 11.58 & $8 \times 10^{-3}$ & 5.5 & $8.38 \times 10^{-4}$ \\
\hline \multirow[t]{5}{*}{30} & 10.86 & $2 \times 10^{-3}$ & 6 & $3.15 \times 10^{-4}$ \\
\hline & 11.02 & $2 \times 10^{-3}$ & 6.2 & $2.33 \times 10^{-4}$ \\
\hline & 11.17 & $3 \times 10^{-3}$ & 5.8 & $5.81 \times 10^{-4}$ \\
\hline & 11.37 & $6 \times 10^{-3}$ & 5.1 & $2.25 \times 10^{-3}$ \\
\hline & 11.57 & $8 \times 10^{-3}$ & 6 & $1.81 \times 10^{-3}$ \\
\hline \multirow[t]{4}{*}{30} & 10.81 & $2 \times 10^{-3}$ & 6.5 & $1.86 \times 10^{-4}$ \\
\hline & 11.06 & $3 \times 10^{-3}$ & 6.3 & $2.25 \times 10^{-4}$ \\
\hline & 11.32 & $6 \times 10^{-3}$ & 6.3 & $8.29 \times 10^{-4}$ \\
\hline & 11.55 & $8 \times 10^{-3}$ & 6.7 & $8.93 \times 10^{-4}$ \\
\hline \multirow[t]{4}{*}{30} & 10.66 & $2 \times 10^{-3}$ & 5.72 & $1.08 \times 10^{-4}$ \\
\hline & 10.85 & $4 \times 10^{-3}$ & 5.58 & $2.43 \times 10^{-4}$ \\
\hline & 11.12 & $6 \times 10^{-3}$ & 5.72 & $3.12 \times 10^{-4}$ \\
\hline & 11.21 & $8 \times 10^{-3}$ & 5.68 & $4.41 \times 10^{-4}$ \\
\hline \multirow[t]{4}{*}{30} & 10.68 & $2 \times 10^{-3}$ & 6.04 & $2.01 \times 10^{-4}$ \\
\hline & 10.88 & $4 \times 10^{-3}$ & 6.05 & $2.96 \times 10^{-4}$ \\
\hline & 11.16 & $6 \times 10^{-3}$ & 5.98 & $4.96 \times 10^{-4}$ \\
\hline & 11.28 & $8 \times 10^{-3}$ & 5.61 & $5.58 \times 10^{-4}$ \\
\hline \multirow[t]{4}{*}{30} & 10.71 & $2 \times 10^{-3}$ & 5.58 & $1.92 \times 10^{-4}$ \\
\hline & 10.91 & $4 \times 10^{-3}$ & 5.56 & $3.24 \times 10^{-4}$ \\
\hline & 11.15 & $6 \times 10^{-3}$ & 5.45 & $3.99 \times 10^{-4}$ \\
\hline & 11.26 & $8 \times 10^{-3}$ & 5.45 & $5.82 \times 10^{-4}$ \\
\hline \multirow[t]{4}{*}{35} & 10.77 & $2 \times 10^{-3}$ & 5.8 & $8.09 \times 10^{-4}$ \\
\hline & 10.94 & $3 \times 10^{-3}$ & 5.9 & $9.64 \times 10^{-4}$ \\
\hline & 11.3 & $6 \times 10^{-3}$ & 5.9 & $2.27 \times 10^{-3}$ \\
\hline & 11.49 & $8 \times 10^{-3}$ & 5.8 & $2.81 \times 10^{-3}$ \\
\hline
\end{tabular}

with increase in $\mathrm{pH}$ (Figure 5).

Since in this reaction, multiple hydrogen sulfide species are involved, $\left[\mathrm{H}^{+}\right]$- dependence is an important parameter. The order of reaction with respect to $\left[\mathrm{H}^{+}\right]$was determined from the plot of $\log k_{\mathrm{obs}}$ versus $\log \left[\mathrm{H}^{+}\right]$and found to be 0.20 . The small fractional negative order in $\left[\mathrm{H}^{+}\right]$indicates the effect of $\mathrm{H}^{+}$ion to be small. Indeed, a change in $\left[\mathrm{H}^{+}\right]$ by an order of three resulted in a change in $k_{\text {obs }}$ by an order of less than one. 


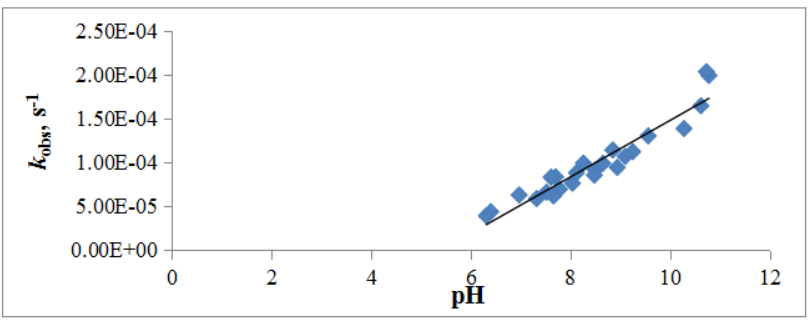

Figure 5. The plot of $k_{\mathrm{obs}}$ versus pH at $[\mathrm{S}]=2 \times 10^{-3} \mathrm{~mol} \mathrm{~L}^{-1}$ and $\mathrm{T}=30^{\circ} \mathrm{C}$

\subsection{Effect of Ammonium Nitrate}

Ammonium aerosols and ammonia gas are important trace atmospheric constituents and, therefore, the effect of ammonium nitrate was examined. The addition of ammonium nitrate had little effect on the reaction rate. This is contrary to findings of dissolved $\mathrm{SO}_{2}$ oxidation in which both ammonia and ammonium ions strongly inhibited $\mathrm{SO}_{2}$ oxidation ${ }^{[64]}$.

\subsection{Energy of Activation}

By determining $k_{\mathrm{obs}}$ at three different temperatures and at $[\mathrm{S}]=2 \times 10^{-3} \mathrm{~mol} \mathrm{~L}^{-1}$, the energy of activation, $\mathrm{E}_{\mathrm{a}}$, was determined to be $28.3 \mathrm{kcal} \mathrm{mol}^{-1}$. Energy of activation is defined as the minimum amount of energy that must be provided to compounds to result in a chemical reaction. In previous studies using highly purified systems, $\mathrm{E}_{\mathrm{a}}$ values have been reported to be $8 \pm 0.4 \mathrm{kcal} \mathrm{mol}^{-1}{ }^{[17]}$ and 14.07 $\mathrm{kcal} \mathrm{mol}^{-1}[19]$. The significant difference in $\mathrm{E}_{\mathrm{a}}$ values appears to be due the difference in reaction conditions employed.

\subsection{Effect of Anthropogenic VOCs}

Variety of volatile organic compounds such as aromatics, alcohols, terpenes, phenols, carbonyl compounds, carboxylic acids, esters, etc. are found as trace constituents in atmosphere. Sources of VOCs are both biogenic and anthropogenic. VOCs are involved

Table 4. The values of $k_{\text {obs }}$ at different $\mathrm{pH}$ at $[\mathrm{S}]=2 \times 10^{-3} \mathrm{~mol} \mathrm{~L}^{-1}$ and $\mathrm{T}=30^{\circ} \mathrm{C}$

\begin{tabular}{|c|c|c|c|c|}
\hline $\mathrm{pH}$ & $\begin{array}{c}k_{\mathrm{obs}}\left\{\left[\mathrm{H}^{+}\right]^{2}+\right. \\
\left.\mathrm{K}_{1}\left[\mathrm{H}^{+}\right]+\mathrm{K}_{1} \mathrm{~K}_{2}\right\}\end{array}$ & $k_{\mathrm{obs}}, \mathrm{s}^{-1}$ & $\begin{array}{c}k_{\mathrm{obs}} /[\mathrm{S}]=k \\
\mathrm{~L} \mathrm{~mol}^{-1} \mathrm{~s}^{-1}\end{array}$ & $\begin{array}{c}k\left\{\left[\mathrm{H}^{+}\right]^{2}+\right. \\
\left.\mathrm{K}_{1}\left[\mathrm{H}^{+}\right]+\mathrm{K}_{1} \mathrm{~K}_{2}\right\}\end{array}$ \\
\hline 10.78 & $3.22 \times 10^{-22}$ & $2.00 \times 10^{-4}$ & $9.98 \times 10^{-2}$ & $1.61 \times 10^{-19}$ \\
\hline 10.73 & $3.69 \times 10^{-22}$ & $2.04 \times 10^{-4}$ & $1.02 \times 10^{-1}$ & $1.84 \times 10^{-19}$ \\
\hline 10.62 & $3.83 \times 10^{-22}$ & $1.65 \times 10^{-4}$ & $8.25 \times 10^{-2}$ & $1.92 \times 10^{-19}$ \\
\hline 10.57 & $1.03 \times 10^{-21}$ & $3.94 \times 10^{-4}$ & $1.97 \times 10^{-1}$ & $5.13 \times 10^{-19}$ \\
\hline 10.48 & $8.04 \times 10^{-22}$ & $2.51 \times 10^{-4}$ & $1.26 \times 10^{-1}$ & $4.02 \times 10^{-19}$ \\
\hline 10.41 & $4.78 \times 10^{-22}$ & $1.27 \times 10^{-4}$ & $6.35 \times 10^{-2}$ & $2.39 \times 10^{-19}$ \\
\hline 10.35 & $2.21 \times 10^{-21}$ & $5.12 \times 10^{-4}$ & $2.56 \times 10^{-1}$ & $1.11 \times 10^{-18}$ \\
\hline 10.34 & $5.74 \times 10^{-22}$ & $1.30 \times 10^{-4}$ & $6.50 \times 10^{-2}$ & $2.87 \times 10^{-19}$ \\
\hline 10.28 & $7.05 \times 10^{-22}$ & $1.39 \times 10^{-4}$ & $6.95 \times 10^{-2}$ & $3.52 \times 10^{-19}$ \\
\hline 10.26 & $7.33 \times 10^{-22}$ & $1.38 \times 10^{-4}$ & $6.90 \times 10^{-2}$ & $3.66 \times 10^{-19}$ \\
\hline 10.13 & $2.37 \times 10^{-21}$ & $3.31 \times 10^{-4}$ & $1.66 \times 10^{-1}$ & $1.18 \times 10^{-18}$ \\
\hline 10.07 & $1.63 \times 10^{-21}$ & $1.98 \times 10^{-4}$ & $9.90 \times 10^{-2}$ & $8.14 \times 10^{-19}$ \\
\hline 10.02 & $3.45 \times 10^{-21}$ & $3.74 \times 10^{-4}$ & $1.87 \times 10^{-1}$ & $1.73 \times 10^{-18}$ \\
\hline 9.96 & $4.24 \times 10^{-21}$ & $4.00 \times 10^{-4}$ & $2.00 \times 10^{-1}$ & $2.12 \times 10^{-18}$ \\
\hline 9.81 & $7.57 \times 10^{-21}$ & $5.06 \times 10^{-4}$ & $2.53 \times 10^{-1}$ & $3.78 \times 10^{-18}$ \\
\hline 9.7 & $4.34 \times 10^{-21}$ & $2.25 \times 10^{-4}$ & $1.13 \times 10^{-1}$ & $2.17 \times 10^{-18}$ \\
\hline 9.56 & $3.47 \times 10^{-21}$ & $1.31 \times 10^{-4}$ & $6.53 \times 10^{-2}$ & $1.74 \times 10^{-18}$ \\
\hline 9.53 & $1.06 \times 10^{-20}$ & $3.72 \times 10^{-4}$ & $1.86 \times 10^{-1}$ & $5.31 \times 10^{-18}$ \\
\hline 9.47 & $1.09 \times 10^{-20}$ & $3.32 \times 10^{-4}$ & $1.66 \times 10^{-1}$ & $5.44 \times 10^{-18}$ \\
\hline 9.34 & $1.55 \times 10^{-20}$ & $3.50 \times 10^{-4}$ & $1.75 \times 10^{-1}$ & $7.74 \times 10^{-18}$ \\
\hline 9.25 & $6.13 \times 10^{-21}$ & $1.12 \times 10^{-4}$ & $5.62 \times 10^{-2}$ & $3.06 \times 10^{-18}$ \\
\hline 9.2 & $3.71 \times 10^{-20}$ & $6.06 \times 10^{-4}$ & $3.03 \times 10^{-1}$ & $1.85 \times 10^{-17}$ \\
\hline 9.11 & $3.90 \times 10^{-20}$ & $5.17 \times 10^{-4}$ & $2.59 \times 10^{-1}$ & $1.95 \times 10^{-17}$ \\
\hline 9.1 & $8.26 \times 10^{-21}$ & $1.07 \times 10^{-4}$ & $5.35 \times 10^{-2}$ & $4.13 \times 10^{-18}$ \\
\hline 9.08 & $3.64 \times 10^{-20}$ & $4.51 \times 10^{-4}$ & $2.26 \times 10^{-1}$ & $1.82 \times 10^{-17}$ \\
\hline 8.94 & $1.06 \times 10^{-20}$ & $9.44 \times 10^{-5}$ & $4.72 \times 10^{-2}$ & $5.28 \times 10^{-18}$ \\
\hline 8.85 & $8.59 \times 10^{-20}$ & $6.22 \times 10^{-4}$ & $3.11 \times 10^{-1}$ & $4.29 \times 10^{-17}$ \\
\hline
\end{tabular}

\begin{tabular}{|c|c|c|c|c|}
\hline $\mathrm{pH}$ & $\begin{array}{c}k_{\mathrm{obs}}\left\{\left[\mathrm{H}^{+}\right]^{2}+\right. \\
\left.\mathrm{K}_{1}\left[\mathrm{H}^{+}\right]+\mathrm{K}_{1} \mathrm{~K}_{2}\right\}\end{array}$ & $k_{\mathrm{obs}}, \mathrm{s}^{-1}$ & $\begin{array}{c}k_{\mathrm{obs}} /[\mathrm{S}]=k \\
\mathrm{~L} \mathrm{~mol}^{-1} \mathrm{~s}^{-1}\end{array}$ & $\begin{array}{c}k\left\{\left[\mathrm{H}^{+}\right]^{2}+\right. \\
\left.\mathrm{K}_{1}\left[\mathrm{H}^{+}\right]+\mathrm{K}_{1} \mathrm{~K}_{2}\right\}\end{array}$ \\
\hline 8.85 & $1.58 \times 10^{-20}$ & $1.14 \times 10^{-4}$ & $5.72 \times 10^{-2}$ & $7.90 \times 10^{-18}$ \\
\hline 8.72 & $1.65 \times 10^{-19}$ & $8.84 \times 10^{-4}$ & $4.42 \times 10^{-1}$ & $8.27 \times 10^{-17}$ \\
\hline 8.71 & $3.85 \times 10^{-20}$ & $2.01 \times 10^{-4}$ & $1.00 \times 10^{-1}$ & $1.92 \times 10^{-17}$ \\
\hline 8.65 & $2.19 \times 10^{-20}$ & $9.90 \times 10^{-5}$ & $4.95 \times 10^{-2}$ & $1.09 \times 10^{-17}$ \\
\hline 8.56 & $5.55 \times 10^{-20}$ & $2.04 \times 10^{-4}$ & $1.02 \times 10^{-1}$ & $2.78 \times 10^{-17}$ \\
\hline 8.5 & $2.89 \times 10^{-20}$ & $9.17 \times 10^{-5}$ & $4.59 \times 10^{-2}$ & $1.44 \times 10^{-17}$ \\
\hline 8.48 & $2.82 \times 10^{-20}$ & $8.56 \times 10^{-5}$ & $4.28 \times 10^{-2}$ & $1.41 \times 10^{-17}$ \\
\hline 8.36 & $9.76 \times 10^{-20}$ & $2.22 \times 10^{-4}$ & $1.11 \times 10^{-1}$ & $4.88 \times 10^{-17}$ \\
\hline 8.26 & $5.56 \times 10^{-20}$ & $9.94 \times 10^{-5}$ & $4.97 \times 10^{-2}$ & $2.78 \times 10^{-17}$ \\
\hline 8.16 & $1.63 \times 10^{-20}$ & $2.28 \times 10^{-5}$ & $1.14 \times 10^{-2}$ & $8.16 \times 10^{-18}$ \\
\hline 8.16 & $6.32 \times 10^{-20}$ & $8.86 \times 10^{-5}$ & $4.43 \times 10^{-2}$ & $3.16 \times 10^{-17}$ \\
\hline 8.12 & $6.96 \times 10^{-20}$ & $8.83 \times 10^{-5}$ & $4.41 \times 10^{-2}$ & $3.48 \times 10^{-17}$ \\
\hline 8.04 & $7.35 \times 10^{-20}$ & $7.64 \times 10^{-5}$ & $3.82 \times 10^{-2}$ & $3.67 \times 10^{-17}$ \\
\hline 7.78 & $1.30 \times 10^{-19}$ & $6.95 \times 10^{-5}$ & $3.47 \times 10^{-2}$ & $6.51 \times 10^{-17}$ \\
\hline 7.7 & $1.94 \times 10^{-19}$ & $8.37 \times 10^{-5}$ & $4.18 \times 10^{-2}$ & $9.71 \times 10^{-17}$ \\
\hline 7.66 & $1.60 \times 10^{-19}$ & $6.18 \times 10^{-5}$ & $3.09 \times 10^{-2}$ & $7.99 \times 10^{-17}$ \\
\hline 7.61 & $2.47 \times 10^{-19}$ & $8.32 \times 10^{-5}$ & $4.16 \times 10^{-2}$ & $1.23 \times 10^{-16}$ \\
\hline 7.52 & $2.52 \times 10^{-19}$ & $6.60 \times 10^{-5}$ & $3.30 \times 10^{-2}$ & $1.26 \times 10^{-16}$ \\
\hline 7.51 & $3.11 \times 10^{-19}$ & $7.91 \times 10^{-5}$ & $3.96 \times 10^{-2}$ & $1.55 \times 10^{-16}$ \\
\hline 7.32 & $4.05 \times 10^{-19}$ & $5.87 \times 10^{-5}$ & $2.94 \times 10^{-2}$ & $2.03 \times 10^{-16}$ \\
\hline 7.28 & $1.66 \times 10^{-19}$ & $2.13 \times 10^{-4}$ & $1.07 \times 10^{-1}$ & $8.32 \times 10^{-16}$ \\
\hline 7.23 & $3.42 \times 10^{-19}$ & $3.75 \times 10^{-5}$ & $1.87 \times 10^{-2}$ & $1.71 \times 10^{-16}$ \\
\hline 6.97 & $1.37 \times 10^{-18}$ & $6.29 \times 10^{-5}$ & $3.15 \times 10^{-2}$ & $6.86 \times 10^{-16}$ \\
\hline 6.4 & $8.61 \times 10^{-18}$ & $4.38 \times 10^{-5}$ & $2.19 \times 10^{-2}$ & $4.31 \times 10^{-15}$ \\
\hline 6.31 & $1.26 \times 10^{-17}$ & $3.92 \times 10^{-5}$ & $1.96 \times 10^{-2}$ & $6.28 \times 10^{-15}$ \\
\hline 5.95 & $2.84 \times 10^{-17}$ & $2.08 \times 10^{-5}$ & $1.04 \times 10^{-2}$ & $1.42 \times 10^{-14}$ \\
\hline
\end{tabular}


in many atmospheric reactions. Chen and Morris ${ }^{[16]}$ examined the effect of some organics and found that, whereas EDTA, nitrilotriacetic acid (NTA), cyanide, peptone, citrate and glycerol inhibit metal catalyzed $\mathrm{H}_{2} \mathrm{~S}$ autoxidation, hydroquinone, formaldehyde and phenol accelerated the autoxidation ${ }^{[11,21,65]}$.

Unlike the work of Chen and Morris ${ }^{[16]}$, we examined the effect of anthropogenic VOCs on $\mathrm{H}_{2} \mathrm{~S}$ oxidation in unbuffered medium and in the absence of any added catalyst. Under these conditions, our results indicate anthropogenic VOCs such as acetanilide, benzene, ethanol, aniline, toluene, benzamide, $o$-xylene, $m$-xylene, $p$-xylene, anisole to have no significant effect on the reaction rate and any observed small effect is within the uncertainty of the rate measurements. The results are shown in Table 5.

Table 5. The selected values of first -order rate constants, $k_{\text {obs }}$, at $30^{\circ} \mathrm{C}$

\begin{tabular}{|c|c|c|c|c|}
\hline Compound & $\mathrm{pH}$ & [VOCs], $\mathrm{mol} \mathrm{L}^{-1}$ & {$[\mathrm{~S}], \mathrm{mol} \mathrm{L}^{-1}$} & $k_{\mathrm{obs}}, \mathrm{s}^{-1}$ \\
\hline \multirow{3}{*}{ Acetanilide } & 10.73 & 0 & $2 \times 10^{-3}$ & $1.65 \times 10^{-4}$ \\
\hline & 10.83 & $3 \times 10^{-4}$ & $2 \times 10^{-3}$ & $1.29 \times 10^{-4}$ \\
\hline & 10.78 & $7 \times 10^{-4}$ & $2 \times 10^{-3}$ & $1.47 \times 10^{-4}$ \\
\hline \multirow{3}{*}{ Benzene } & 10.73 & 0 & $2 \times 10^{-3}$ & $1.36 \times 10^{-4}$ \\
\hline & 10.78 & $2 \times 10^{-4}$ & $2 \times 10^{-3}$ & $1.16 \times 10^{-4}$ \\
\hline & 10.81 & $3 \times 10^{-4}$ & $2 \times 10^{-3}$ & $1.26 \times 10^{-4}$ \\
\hline \multirow{4}{*}{ Toluene } & 10.8 & 0 & $2 \times 10^{-3}$ & $2.27 \times 10^{-4}$ \\
\hline & 10.88 & $3 \times 10^{-4}$ & $2 \times 10^{-3}$ & $1.37 \times 10^{-4}$ \\
\hline & 10.88 & $5 \times 10^{-4}$ & $2 \times 10^{-3}$ & $1.29 \times 10^{-4}$ \\
\hline & 10.88 & $8 \times 10^{-4}$ & $2 \times 10^{-3}$ & $1.80 \times 10^{-4}$ \\
\hline \multirow{3}{*}{ Ethanol } & 10.78 & 0 & $2 \times 10^{-3}$ & $1.30 \times 10^{-4}$ \\
\hline & 10.8 & $4 \times 10^{-5}$ & $2 \times 10^{-3}$ & $1.16 \times 10^{-4}$ \\
\hline & 10.8 & $6 \times 10^{-5}$ & $2 \times 10^{-3}$ & $1.15 \times 10^{-4}$ \\
\hline \multirow{3}{*}{ Aniline } & 10.8 & 0 & $2 \times 10^{-3}$ & $1.02 \times 10^{-4}$ \\
\hline & 10.8 & $3 \times 10^{-4}$ & $2 \times 10^{-3}$ & $1.45 \times 10^{-4}$ \\
\hline & 10.81 & $6 \times 10^{-4}$ & $2 \times 10^{-3}$ & $1.41 \times 10^{-4}$ \\
\hline \multirow{3}{*}{ Anisole } & 10.72 & 0 & $2 \times 10^{-3}$ & $1.78 \times 10^{-4}$ \\
\hline & 10.73 & $5 \times 10^{-5}$ & $2 \times 10^{-3}$ & $1.78 \times 10^{-4}$ \\
\hline & 10.73 & $1 \times 10^{-4}$ & $2 \times 10^{-3}$ & $1.39 \times 10^{-4}$ \\
\hline \multirow{3}{*}{$o$-xylene } & 10.75 & 0 & $2 \times 10^{-3}$ & $1.35 \times 10^{-4}$ \\
\hline & 10.74 & $2 \times 10^{-5}$ & $2 \times 10^{-3}$ & $1.55 \times 10^{-4}$ \\
\hline & 10.74 & $2 \times 10^{-4}$ & $2 \times 10^{-3}$ & $1.72 \times 10^{-4}$ \\
\hline \multirow{3}{*}{$m$-xylene } & 10.74 & 0 & $2 \times 10^{-3}$ & $2.14 \times 10^{-4}$ \\
\hline & 10.73 & $5 \times 10^{-5}$ & $2 \times 10^{-3}$ & $2.67 \times 10^{-4}$ \\
\hline & 10.74 & $1 \times 10^{-4}$ & $2 \times 10^{-3}$ & $2.60 \times 10^{-4}$ \\
\hline \multirow{3}{*}{$p$-xylene } & 10.75 & 0 & $2 \times 10^{-3}$ & $2.84 \times 10^{-4}$ \\
\hline & 10.75 & $5 \times 10^{-5}$ & $2 \times 10^{-3}$ & $2.41 \times 10^{-4}$ \\
\hline & 10.74 & $1 \times 10^{-4}$ & $2 \times 10^{-3}$ & $2.65 \times 10^{-4}$ \\
\hline \multirow{2}{*}{ Benzamide } & 10.75 & 0 & $2 \times 10^{-3}$ & $0.80 \times 10^{-4}$ \\
\hline & 10.74 & $2 \times 10^{-4}$ & $2 \times 10^{-3}$ & $0.72 \times 10^{-4}$ \\
\hline
\end{tabular}

\section{Discussion}

The equilibria (6-7) govern the dissociation of $\mathrm{H}_{2} \mathrm{~S}$ :

$\mathrm{H}_{2} \mathrm{~S} \stackrel{\mathrm{K}_{1}}{\rightleftharpoons} \mathrm{HS}^{-}+\mathrm{H}^{+}$

$\mathrm{HS}^{-} \stackrel{\mathrm{K}_{2}}{\rightleftharpoons} \mathrm{S}^{2-}+\mathrm{H}^{+}$

Where $\mathrm{K}_{1}$ and $\mathrm{K}_{2}$ are first and second dissociation constants of $\mathrm{H}_{2} \mathrm{~S}$. The kinetics in this work was investigated in the $\mathrm{pH}$ range 6.3- 10.8 and $\mathrm{K}_{1}=9.63 \times 10^{-8}$ and $\mathrm{K}_{2}=1.34 \times 10^{-13}$ were used ${ }^{[66]}$.

A speciation study showed that in aqueous media below $\mathrm{pH} 7$, the dominant form shall be $\mathrm{H}_{2} \mathrm{~S}$. On the other hand in the $\mathrm{pH}$ range 7-11, $\mathrm{HS}^{-}$shall be dominant. Species $\mathrm{S}^{2-}$ would become important only beyond $\mathrm{pH} 11.5^{[38,67]}$. This indicates that under our experimental conditions the major reactive species is expected to be $\mathrm{HS}^{-[17]}$. Chen and Morris ${ }^{[16]}$ have shown that $\mathrm{HS}^{-}$is the dominant species in sulfide solutions everywhere from $\mathrm{pH} 7$ to $\mathrm{pH} 12.5$ and that it comprises better than $90 \%$ of the all sulfide species in aqueous solution from $\mathrm{pH} 8$ to 11.5. Likewise, Millero ${ }^{[21]}$ has shown that at $\mathrm{pH} 8.1$ in aqueous solution $\left[\mathrm{H}_{2} \mathrm{~S}\right]=$ $7.05 \%,\left[\mathrm{HS}^{-}\right]=92.95 \%$ and $\left[\mathrm{S}^{2-}\right]=2.9 \times 10^{-5} \%$. Lewis ${ }^{[68]}$ plotted the concentration of sulfide species against $\mathrm{pH}$ and showed HS ${ }^{-}$to be dominant species between $\mathrm{pH} 7$-14.

The oxidation of the three principal species $\mathrm{H}_{2} \mathrm{~S}$, HS and $\mathrm{S}^{2-}$ by oxygen is possible. These species are expected to be reactive but at relatively different rates of oxidation. Chen and Morris ${ }^{[16]}$ reported that in acid solutions, $\mathrm{pH}<6$, where $\mathrm{H}_{2} \mathrm{~S}$ is the predominant sulfide species, the rate of oxidation is very low. When the $\mathrm{pH}$ was increased, the rate also increased, showing that $\mathrm{HS}^{-}$is much more reactive than $\mathrm{H}_{2} \mathrm{~S}$ species. This work also showed the $\mathrm{S}^{2-}$ species to be less reactive than HS species ${ }^{[16]}$. The reaction has been studied by Avrahami and Golding ${ }^{[17]}$ at very low $\mathrm{S}^{2-}$ concentrations in the $\mathrm{pH}$ range 11-13 and they explained the kinetics by assuming HS to be reactive. Likewise, Luther et al. ${ }^{[37]}$ also assumed $\mathrm{HS}^{-}$to be reactive species to explain their results in the $\mathrm{pH} 8-12$. All these results indicate that in the oxidation of aquated hydrogen sulfide by $\mathrm{O}_{2}$, the dominant and reactive species is $\mathrm{HS}^{-}$in the $\mathrm{pH}$ range 7-11.

Now we turn our attention to results of $\mathrm{pH}$ dependence of this work. Since all the three species $\mathrm{H}_{2} \mathrm{~S}, \mathrm{HS}^{-}$and $\mathrm{S}^{2-}$ formed are governed by the equilibria (6) and (7), assuming all the three species to be reactive a following simplified general mechanism of hydrogen sulfide, [S(-II)], for its oxidation by $\mathrm{O}_{2}$ may be written.

$\mathrm{H}_{2} \mathrm{~S}+\mathrm{O}_{2} \stackrel{k_{0}}{\longrightarrow}$ Products
$\mathrm{HS}^{-}+\mathrm{O}_{2} \stackrel{k_{1}}{\longrightarrow}$ Products
$\mathrm{S}^{2-}+\mathrm{O}_{2} \stackrel{k_{2}}{\longrightarrow}$ Products


Based on the mechanism (8-10), the general rate law (11) can be derived.

$$
-\mathrm{d}\left[\mathrm{O}_{2}\right] / \mathrm{dt}=k_{0}\left[\mathrm{H}_{2} \mathrm{~S}\right]\left[\mathrm{O}_{2}\right]+k_{1}\left[\mathrm{HS}^{-}\right]\left[\mathrm{O}_{2}\right]+k_{2}\left[\mathrm{~S}^{2-}\right]\left[\mathrm{O}_{2}\right]
$$

Equations (5-11) lead to the rate law (12):

$$
\frac{-\mathrm{d}\left[\mathrm{O}_{2}\right]}{\mathrm{dt}}=\frac{[\mathrm{S}]\left[\mathrm{O}_{2}\right] \mathrm{t}\left(k_{0}\left[\mathrm{H}_{+}\right]_{2}+k_{1} \mathrm{~K}_{1}\left[\mathrm{H}_{+}\right]+k_{2} \mathrm{~K}_{1} \mathrm{~K}_{2}\right)}{\left(\left[\mathrm{H}_{+}\right]_{2}+\mathrm{K}_{1}\left[\mathrm{H}_{+}\right]+\mathrm{K}_{1} \mathrm{~K}_{2}\right)}
$$

The rate law (12) predicts that the plot of $k_{\mathrm{obs}}$ versus $\left[\mathrm{H}^{+}\right]$ should be non-linear, which was actually found to be true.

In this study, which was carried out in the $\mathrm{pH}$ range 6-10.5, the species present largely would be $\mathrm{H}_{2} \mathrm{~S}$ and $\mathrm{HS}^{-}$ with the latter being dominant. A detailed analysis of the $\left[\mathrm{H}^{+}\right]$-dependence indicated only $\mathrm{HS}^{-}$to be reactive. Hence, when only $\mathrm{HS}^{-}$is considered to be reactive and dominant, the Equation (12) is modified to Equation (13).

$$
\frac{-\mathrm{d}\left[\mathrm{O}_{2}\right]}{\mathrm{dt}}=\frac{k_{1} \mathrm{~K}_{1}\left[\mathrm{H}_{+}\right]\left[\mathrm{S}_{0}\right]\left[\mathrm{O}_{2}\right]_{\mathrm{t}}}{\left(\left[\mathrm{H}_{+}\right]_{2}+\mathrm{K}_{1}[\mathrm{H}+]+\mathrm{K}_{1} \mathrm{~K}_{2}\right)}
$$

On comparing Equations (5) and (13), we get Equation (14), which rearranges to Equation (15).

$$
\begin{aligned}
& k=\frac{k_{1} \mathrm{~K}_{1}\left[\mathrm{H}_{+}\right]}{\left(\left[\mathrm{H}_{+}\right]_{2}+\mathrm{K}_{1}\left[\mathrm{H}_{+}\right]+\mathrm{K}_{1} \mathrm{~K}_{2}\right)} \\
& k\left(\left[\mathrm{H}^{+}\right]^{2}+\mathrm{K}_{1}\left[\mathrm{H}^{+}\right]+\mathrm{K}_{1} \mathrm{~K}_{2}\right)=k_{1} \mathrm{~K}_{1}\left[\mathrm{H}^{+}\right]
\end{aligned}
$$

As required by Equation (15), the plot between $k\left(\left[\mathrm{H}^{+}\right]^{2}\right.$ $+\mathrm{K}_{1}\left[\mathrm{H}^{+}\right]+\mathrm{K}_{1} \mathrm{~K}_{2}$ ) and $\left[\mathrm{H}^{+}\right]$was linear (Figure 6). Since $\left[\mathrm{H}^{+}\right]$dependence is important in deciding the reactive S(-II) species, more than seventy experiments were performed at $30^{\circ} \mathrm{C}$ and at different $\mathrm{pH}$ values keeping all other parameters constant. These $k_{\text {obs }}$ values were used in drawing the plot between $k\left(\left[\mathrm{H}^{+}\right]^{2}+\mathrm{K}_{1}\left[\mathrm{H}^{+}\right]+\mathrm{K}_{1} \mathrm{~K}_{2}\right)$ and $\left[\mathrm{H}^{+}\right]$, which was a straight line passing through origin(Figure 6). The values of parameter, $k\left(\left[\mathrm{H}^{+}\right]^{2}+\right.$ $\mathrm{K}_{1}\left[\mathrm{H}^{+}\right]+\mathrm{K}_{1} \mathrm{~K}_{2}$ ), at different $\left[\mathrm{H}^{+}\right]$are collected in Table 4. According to Equation (15), the slope is equal to $k_{1} \mathrm{~K}_{1}$ (Figure 6), which on division by $\mathrm{K}_{1}$ yielded the value of $k_{1}$. From the value of slope $(1.25 \pm 0.02) \times 10^{-8}$, using $\mathrm{K}_{1}=9.63 \times 10^{-8}$ the value of $k_{1}$ was found to be $0.13 \mathrm{~L} \mathrm{~mol}^{-}$
${ }^{1} \mathrm{~s}^{-1}$ at $\mathrm{t}=30^{\circ} \mathrm{C}$.

In the region of $\mathrm{pH}$ (7-11) generally used in this study, the reaction of $\mathrm{O}_{2}$ with $\mathrm{HS}^{-}$may occur through either a one-electron transfer reaction (16) or a two-electron transfer reaction(17).

$\mathrm{O}_{2(\mathrm{aq})}+\mathrm{HS}^{-} \longrightarrow \mathrm{O}_{2(\mathrm{aq})}^{-}+\mathrm{HS}$

$\mathrm{O}_{2}+\mathrm{H}_{2} \mathrm{~S} \longrightarrow \mathrm{S}^{0}+\mathrm{H}_{2} \mathrm{O}_{2}$

Thermodynamically, one-electron transfer and twoelectron transfer reactions have been shown to be unfavorable and favorable, respectively and, therefore, the formation of superoxide ion, $\mathrm{O}_{2}^{-}$, and bisulfide radical, HS, has been considered unlikely ${ }^{[37]}$. It has been pointed out that sulfur $\left(\mathrm{S}^{0}\right)$ combines with sulfide ion to form polysulfide $\left(\mathrm{S}_{\mathrm{x}}{ }^{2-}\right)(\mathrm{x}=2-5)$ ions $(18)$, but when $\mathrm{pH}>7.5$ sulfide has a higher holding capacity for sulfur ${ }^{[16]}$. Thus, the precipitation of sulfur becomes less likely: indeed, we found no precipitation of colloidal sulfur.

$\mathrm{HS}^{-}+(\mathrm{x}-1) \mathrm{S} \longrightarrow \mathrm{S}_{\mathrm{x}}^{2-}+\mathrm{H}^{+}$

Chen and Morris ${ }^{[16]}$ suggested that the ultimate product of $\mathrm{HS}^{-}$and $\mathrm{O}_{2}$ is sulfate after passing through several intermediates.

For this autoxidation reaction, many diverse speculative mechanisms have been proposed ${ }^{[11,16,59,60,69]}$. These mechanisms fall in two distinct kinds of detailed oxidation mechanisms- polar and chain mechanisms. Polar mechanism suggested by Hoffmann and $\mathrm{Lim}^{\left[{ }^{[70]} \text { included }\right.}$ the intermediates $\mathrm{HSO}_{2}^{-}, \mathrm{O}_{2}^{-}, \mathrm{HSO}_{3}^{-}, \mathrm{HO}_{2}, \mathrm{H}_{2} \mathrm{O}_{2}$, etc. The formation of sulfate comes from the oxidation of sulfite with oxygen. On the other hand a free radical mechanism involves metal ion as catalyst and several intermediates ${ }^{[60]}$.

Before discussing the mechanism for sulfide $-\mathrm{O}_{2}$ reaction, it is important to point out that this reaction is affected by traces of catalytic metals present in the reagent solutions even when no catalyst is added from outside. For this reason, the autoxidation reaction is characterized by poor reproducibility of reaction rate ${ }^{[59]}$. The involvement of trace

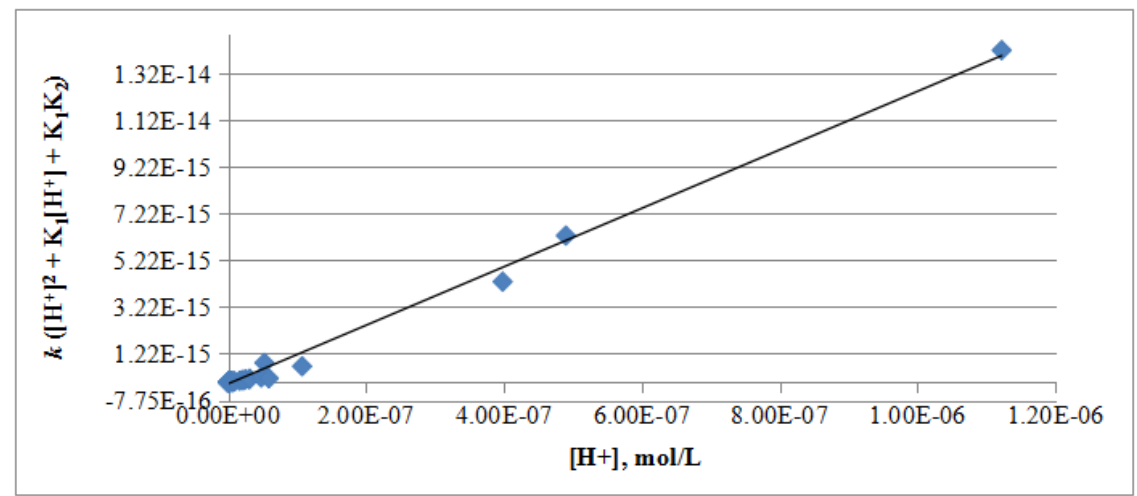

Figure 6. The plot of $k\left\{\left[\mathrm{H}^{+}\right]^{2}+\mathrm{K}_{1}\left[\mathrm{H}^{+}\right]+\mathrm{K}_{1} \mathrm{~K}_{2}\right\}$ versus $\left[\mathrm{H}^{+}\right]$at $[\mathrm{S}]=2 \times 10^{-3} \mathrm{~mol} \mathrm{~L}^{-1}$ and $\mathrm{T}=30^{\circ} \mathrm{C}$ 
metal catalysts has been indicated by decrease in the rate of the reaction by EDTA and other chelating agents ${ }^{[11]}$. We too found strong inhibiting effect of EDTA (Figure 3). This indicates the involvement of trace metal ions as catalysts in any proposed mechanism. The catalytic role of metal ions in the mechanism can be explained as follows ${ }^{[21,65]}$.

$$
\begin{aligned}
& \mathrm{M}^{2+}+\mathrm{O}_{2}+\mathrm{H}^{+} \longrightarrow \mathrm{M}^{3+}+\mathrm{HO}_{2} \\
& \mathrm{M}^{3+}+\mathrm{HS}^{-} \longrightarrow \mathrm{M}^{2+}+\mathrm{HS} \\
& \stackrel{\text { or }}{ }{ }^{\mathrm{M}(\mathrm{HS})^{+}}+\mathrm{O}_{2} \longrightarrow \mathrm{M}^{2+}+\mathrm{S}+\mathrm{HO}_{2}^{-} \\
& \mathrm{HO}_{2}^{-}+\mathrm{HS}^{-} \longrightarrow 2 \mathrm{OH}^{-}+\mathrm{S}
\end{aligned}
$$

The following features of the present study deserve a consideration in proposing any mechanism. One, the strong inhibition of the reaction rate by EDTA suggests the formation of a binary or a ternary complex involving trace metal ions $\mathrm{M}^{\mathrm{n}+}$, present as impurity, and ligands, $\mathrm{O}_{2}$ and/or $\mathrm{HS}^{-}$as in case of Co(II)-4,4',4",4"'tetrasulfophthalocyanine (Co(II)TSP) catalyzed HS autoxidation ${ }^{[59]}$. Two, the effect of VOCs such as benzene, toluene etc., which, are known to strongly inhibit sulfite autoxidation by scavenging intermediates free radicals, is not significant. It suggests that no such free radicals, which can we scavenged by these anthropogenic VOCs are formed and participate in the reaction.

In many proposed mechanisms intermediate $\mathrm{O}_{2}^{-}$has been proposed ${ }^{[60,70]}$. A literature search revealed that the value of the rate constants for the reaction (23) to be $1.5 \times 10^{6} \mathrm{~L} \mathrm{~mol}^{-1} \mathrm{~s}^{-1}$ at $\mathrm{pH} 7.8^{[71]}$.

$\mathrm{HO}_{2} / \mathrm{O}_{2}^{-}+\mathrm{S}^{2-} \longrightarrow$ Products

Although, the concentrations of species $\mathrm{HO}_{2} / \mathrm{O}_{2}^{-}$would be very small, and this combined with the low value of rate constants (23), their role is unlikely to be significant. However, there does exist a possibility of coordinated $\mathrm{HO}_{2} / \mathrm{O}_{2}^{-}$to be much more reactive than the uncoordinated ones, and then these may contribute significantly.

Our attempt failed to find the rate constants of radicals $\mathrm{HO}_{2} / \mathrm{O}_{2}{ }^{-}$with any anthropogenic VOCs studied here and this rules out their inhibitory action as noted by us. This is in contrast to sulfite autoxidation in which the anthropogenic VOCs scavenge the sulfoxy radicals ${ }^{[40,41]}$ and strongly inhibit the oxidation.

The oxidation of $\mathrm{H}_{2} \mathrm{~S}$ by $\mathrm{O}_{2}$ appears to proceed via the formation of a tertiary activated complex in which $\mathrm{O}_{2}$ and $\mathrm{HS}^{-}$are reversibly bound to the metal complex ${ }^{[59]}$ as in the mechanism (24-27).

$$
\begin{aligned}
& \mathrm{M}^{\mathrm{n}+}+\mathrm{O}_{2} \stackrel{\mathrm{K}_{3}}{\rightleftharpoons}\left[\mathrm{MO}_{2}\right]^{\mathrm{n}+} \text { (rapid) } \\
& {\left[\mathrm{MO}_{2}\right]^{\mathrm{n}+}+\mathrm{HS}^{-} \stackrel{\mathrm{K}_{4}}{\rightleftharpoons}\left[\mathrm{M}\left(\mathrm{O}_{2}\right)(\mathrm{HS})\right]^{(\mathrm{n}-1)+} \text { (rapid) }} \\
& {\left[\mathrm{M}\left(\mathrm{O}_{2}\right)(\mathrm{HS})\right]^{(\mathrm{n}-1)+} \stackrel{k_{4}}{\longrightarrow} \mathrm{M}^{\mathrm{n}+}+\mathrm{HSO}_{2}^{-} \text {(slow) }}
\end{aligned}
$$

$\mathrm{HSO}_{2}^{-}+\mathrm{O}_{2} \longrightarrow \mathrm{HSO}_{4}^{-}$(fast)

This mechanism is not unique and can be written in several alternative forms. There is a good possibility of involvement of ternary $\mathrm{M}^{\mathrm{n}+} / \mathrm{M}^{(\mathrm{n}-1)+}-\mathrm{O}_{2}$ - HS ${ }^{-}$complexes and the catalytic role through transfer of electron via $\mathrm{M}^{\mathrm{n}+}$ $\mathrm{M}^{(\mathrm{n}-1)+}$ cycle in this mechanism. This is akin to catalysis of the autoxidation of S(IV) by Co(II)TSP, which was characterized in terms of an ordered ternary-complex mechanism in which both of S(IV) and oxygen were bound to the $\mathrm{Co}(\mathrm{II}) / \mathrm{Co}(\mathrm{III})$ metal center ${ }^{[72]}$.

The complete rate law may be derived as follows. The rate of the reaction is given by Equation (28).

$-\mathrm{d}\left[\mathrm{O}_{2}\right] / \mathrm{dt}=k_{4}\left[\mathrm{M}\left(\mathrm{O}_{2}\right)(\mathrm{HS})\right]^{(\mathrm{n}-1)+}$

From Equation (25), the concentration of the complex $\left[\mathrm{M}\left(\mathrm{O}_{2}\right)(\mathrm{HS})\right]^{(\mathrm{n}-1)+}$ is given by Equation (29).

$\left[\mathrm{M}\left(\mathrm{O}_{2}\right)(\mathrm{HS})\right]^{(\mathrm{n}-1)+}=\mathrm{K}_{4}\left[\mathrm{MO}_{2}\right]^{\mathrm{n}+}\left[\mathrm{HS}^{-}\right]$

On substituting the value of $\left[\mathrm{M}\left(\mathrm{O}_{2}\right)(\mathrm{HS})\right]^{(\mathrm{n}-1)+}$ in Equation (28), we get Equation (30).

$-\mathrm{d}\left[\mathrm{O}_{2}\right] / \mathrm{dt}=k_{4} \mathrm{~K}_{4}\left[\mathrm{MO}_{2}\right]^{\mathrm{n+}}\left[\mathrm{HS}^{-}\right]$

From Equation (24), we have, the concentration of the complex $\left[\mathrm{MO}_{2}\right]^{\mathrm{nt}}$ as in Equation (31).

$\left[\mathrm{MO}_{2}\right]^{\mathrm{n}+}=\mathrm{K}_{3}\left[\mathrm{M}^{\mathrm{n}+}\right]\left[\mathrm{O}_{2}\right]$

From Equations (30) and (31), we get the rate law (32).

$-\mathrm{d}\left[\mathrm{O}_{2}\right] / \mathrm{dt}=k_{4} \mathrm{~K}_{3} \mathrm{~K}_{4}\left[\mathrm{M}^{\mathrm{n}+}\right]\left[\mathrm{O}_{2}\right]\left[\mathrm{HS}^{-}\right]$

Since $\left[\mathrm{HS}^{-}\right]$is part of complex $\left[\mathrm{M}\left(\mathrm{O}_{2}\right)(\mathrm{HS})\right]^{(\mathrm{n}-1)+}$, the total [S] now would be given by Equation (33).

$[\mathrm{S}]=\left[\mathrm{H}_{2} \mathrm{~S}\right]+\left[\mathrm{HS}^{-}\right]+\left[\mathrm{S}^{2-}\right]+\left[\mathrm{M}\left(\mathrm{O}_{2}\right)(\mathrm{HS})\right]^{(\mathrm{n}-1)+}$

And the equilibrium concentration of $\mathrm{HS}^{-}$is given by Equation (34).

$\left[\mathrm{HS}^{-}\right]=\frac{\mathrm{K}_{1}[\mathrm{~S}]\left[\mathrm{H}_{+}\right]}{\left\{\left[\mathrm{H}_{+}\right]_{2}+\mathrm{K}_{1}\left[\mathrm{H}_{+}\right]+\mathrm{K}_{1} \mathrm{~K}_{2}+\mathrm{K}_{1} \mathrm{~K}_{3} \mathrm{~K}_{4}\left[\mathrm{Mn}_{\mathrm{n}}\right]\left[\mathrm{O}_{2}\right]\left[\mathrm{H}_{+}\right]\right\}}$

On combining Equations (32) and (34), we get the complete rate law (35).

$-\mathrm{d}\left[\mathrm{O}_{2}\right] / \mathrm{dt}=\frac{k_{4} \mathrm{~K}_{1} \mathrm{~K}_{3} \mathrm{~K}_{4}\left[\mathrm{Mn}_{\mathrm{n}}\right]\left[\mathrm{O}_{2}\right][\mathrm{S}]\left[\mathrm{H}_{+}\right]}{\left\{\left[\mathrm{H}_{+}\right]_{2}+\mathrm{K}_{1}\left[\mathrm{H}_{+}\right]+\mathrm{K}_{1} \mathrm{~K}_{2}+\mathrm{K}_{1} \mathrm{~K}_{3} \mathrm{~K}_{4}\left[\mathrm{M}_{\mathrm{n}}\right]\left[\mathrm{O}_{2}\right][\mathrm{H}+]\right\}}$

Since the reaction shows a first order dependence in each of $\left[\mathrm{O}_{2}\right]$ and $[\mathrm{HS}-]$, it appears that the term $\left(\mathrm{K}_{1} \mathrm{~K}_{3} \mathrm{~K}_{4}\left[\mathrm{M}^{\mathrm{nt}}\right]\left[\mathrm{O}_{2}\right]\right.$ $\left.\left[\mathrm{H}^{+}\right]\right)$is much smaller than $\left(\left[\mathrm{H}^{+}\right]^{2}+\mathrm{K}_{1}\left[\mathrm{H}^{+}\right]+\mathrm{K}_{1} \mathrm{~K}_{2}\right)$. On ignoring the term $\mathrm{K}_{1} \mathrm{~K}_{3} \mathrm{~K}_{4}\left[\mathrm{M}^{\mathrm{n}}\right]\left[\mathrm{O}_{2}\right]\left[\mathrm{H}^{+}\right]$in the denominator, the rate law (35) modifies to rate law (36).

$-\mathrm{d}\left[\mathrm{O}_{2}\right] / \mathrm{dt}=\frac{k 4 \mathrm{~K}_{1} \mathrm{~K}_{3} \mathrm{~K}_{4}\left[\mathrm{Mn}^{+}\right]\left[\mathrm{O}_{2}\right][\mathrm{S}][\mathrm{H}+]}{\left\{\left[\mathrm{H}_{+}\right]_{2}+\mathrm{K}_{1}\left[\mathrm{H}^{+}\right]+\mathrm{K}_{1} \mathrm{~K}_{2}\right\}}$

The rate law (36) is same as experimental rate law (13) through $k_{1} \mathrm{~K}_{1}=k_{4} \mathrm{~K}_{1} \mathrm{~K}_{3} \mathrm{~K}_{4}\left[\mathrm{M}^{\mathrm{n}+}\right]$.

\subsection{Comparison with Previous Studies}

There have been several kinetics studies on aquated $\mathrm{H}_{2} \mathrm{~S}$ autoxidation. In general, most of the studies including 
the present one support the operation of rate law (13):

$\frac{-\mathrm{d}\left[\mathrm{O}_{2}\right]}{\mathrm{dt}}=\frac{k_{1} \mathrm{~K}_{1}[\mathrm{H}+][\mathrm{S}]\left[\mathrm{O}_{2}\right] \mathrm{t}}{\left(\left[\mathrm{H}_{+}\right]_{2}+\mathrm{K}_{1}[\mathrm{H}+]+\mathrm{K}_{1} \mathrm{~K}_{2}\right)}$

with $\mathrm{HS}^{-}$being the dominant and reactive $\mathrm{H}_{2} \mathrm{~S}$ species in the $\mathrm{pH}$ range(7-11).

It is interesting to compare the $k$ values obtained in these studies (Table 6). The rate constants are under widely different conditions and are based on the different rate laws. Even then the rate constants are not much different $\left(10^{-1}-10^{-2} \mathrm{~L} \mathrm{~mol}^{-1} \mathrm{~s}^{-1}\right)$, although the waters of different types were used. As in previous studies, EDTA ${ }^{[1]}$ inhibited the reaction strongly indicating the involvement of catalytic role of trace metal ions. The effect of organics is small as indicated earlier ${ }^{[11,16,21]}$. Although, free radicals species have been involved by previous workers ${ }^{[60]}$, our studies found no indication of their involvement. We also did not observe any induction period and formation of any colloidal sulfur.

Table 6. The collection of rate constants, $k$, values

\begin{tabular}{|c|c|c|c|}
\hline Rate law & $\begin{array}{c}\text { Rate } \\
\text { constants, } k\end{array}$ & Conditions & Reference \\
\hline \multirow{2}{*}{$\begin{array}{c}-\mathrm{d}\left[\mathrm{S}^{2-}\right] / \mathrm{dt}_{\mathrm{t}=0}=k\left[\mathrm{H}_{2} \mathrm{~S}\right]^{1.34} \\
{\left[\mathrm{O}_{2}\right]^{0.56}}\end{array}$} & $\begin{array}{l}0.38 \times 10^{-2} \\
\mathrm{M}^{-0.9} \mathrm{~s}^{-1}\end{array}$ & $\begin{array}{c}\mathrm{pH}=7.2, \\
\mathrm{O}_{2}=8 \times 10^{-4} \\
25^{\circ} \mathrm{C}\end{array}$ & \multirow{2}{*}[16]{} \\
\hline & $\begin{array}{c}0.41 \times 10^{-2} \\
\mathrm{M}^{-0.9} \mathrm{~s}^{-1}\end{array}$ & $\begin{array}{c}\mathrm{pH}=10.3 \\
\mathrm{O}_{2}=3.2 \times 10^{-4}\end{array}$ & \\
\hline$-\mathrm{d}\left[\mathrm{H}_{2} \mathrm{~S}\right] / \mathrm{dt}=k_{2}\left[\mathrm{H}_{2} \mathrm{~S}\right]\left[\mathrm{O}_{2}\right]$ & $\begin{array}{l}0.19 \times 10^{-2} \\
\mathrm{~L} \mathrm{~mol}^{-1} \mathrm{~s}^{-1}\end{array}$ & $\mathrm{pH}=12,25^{\circ} \mathrm{C}$ & {$[37]$} \\
\hline$-\mathrm{d}\left[\mathrm{H}_{2} \mathrm{~S}\right] / \mathrm{dt}=k_{0}\left[\mathrm{H}_{2} \mathrm{~S}\right]\left[\mathrm{O}_{2}\right]$ & $\begin{array}{l}(0 \pm 2.78) \\
\times \quad 10^{-6} \\
\mathrm{~L} \mathrm{~mol}^{-1} \mathrm{~s}^{-1}\end{array}$ & $\mathrm{pH}=6-8,25^{\circ} \mathrm{C}$ & {$[21]$} \\
\hline$-\mathrm{d}\left[\mathrm{H}_{2} \mathrm{~S}\right] / \mathrm{dt}=k_{2}\left[\mathrm{H}_{2} \mathrm{~S}\right]_{\mathrm{t}}\left[\mathrm{O}_{2}\right]_{\mathrm{t}}$ & $\begin{array}{c}\left(3.5 \times 10^{-6}-\right. \\
\left.0.14 \times 10^{-3}\right) \\
\mathrm{s}^{-1}\end{array}$ & $\begin{array}{c}\mathrm{pH}=4.27-8.01, \\
\quad 28^{\circ} \mathrm{C}, \\
\mathrm{O}_{2}=5-16.4 \mathrm{mgL}^{1}, \\
\quad \text { (Seawater) }\end{array}$ & {$[38]$} \\
\hline$-\mathrm{d}\left[\mathrm{O}_{2}\right] / \mathrm{dt}=k_{1}\left[\mathrm{HS}^{-}\right]\left[\mathrm{O}_{2}\right]$ & $\begin{array}{l}k_{1}=1.3 \times 10^{-} \\
{ }^{1} \mathrm{~L} \mathrm{~mol}^{-1} \mathrm{~s}^{-1}\end{array}$ & $\begin{array}{l}\text { Distilled water, } \\
\mathrm{pH}=6-11,30^{\circ} \mathrm{C}\end{array}$ & This work \\
\hline
\end{tabular}

\subsection{Comparison with $\mathrm{SO}_{2}$ Oxidation}

The chemical behavior of acid rain precursors $\mathrm{SO}_{2}$ and $\mathrm{H}_{2} \mathrm{~S}$ towards the oxidation by atmospheric oxygen is quite different although $\mathrm{H}_{2} \mathrm{~S}$ is believed to be a precursor of $\mathrm{SO}_{2}$. There are some kinetics and mechanistic features, which differentiates their autoxidation.

(1) The kinetics of oxidation of aqueous S(IV) at constant $\mathrm{pH}$ is characterize by the rate law:

$-\mathrm{d}[\mathrm{S}(\mathrm{IV})] / \mathrm{dt}=k[\mathrm{~S}(\mathrm{IV})]$

which is independent of $\left[\mathrm{O}_{2}\right]$.
On the contrary, $\mathrm{H}_{2} \mathrm{~S}$ reaction at constant $\mathrm{pH}$ follows the rate law (5):

$-\mathrm{d}\left[\mathrm{O}_{2}\right] / \mathrm{dt}=k[\mathrm{~S}]\left[\mathrm{O}_{2}\right]_{\mathrm{t}}$

which is first order in $\mathrm{O}_{2}$.

(2) EDTA inhibition of both reactions indicates a catalytic role for trace metal ions.

(3) A big difference in two studies is the effect of organic free radical scavengers. Whereas, S(IV) reaction is strongly inhibited due to scavenging of sulfate radical anion, $\mathrm{SO}_{4}{ }^{-[40,41]}, \mathrm{H}_{2} \mathrm{~S}$ oxidation is not affected significantly. The latter observation does not require the invocation of free radicals in the present study.

\subsection{Application to Atmospheric Chemistry}

The results of this study indicate the oxidation of $\mathrm{H}_{2} \mathrm{~S}$ to be significant in atmospheric aqueous systems and its atmospheric aqueous phase conversion rates were calculated using Equation (37) ${ }^{[61]}$.

Conversion rate $\left(\%\right.$ day $\left.^{-1}\right)=$

$\left[\frac{\left\{100 k\left[\mathrm{O}_{2}\right][\mathrm{S}] \mathrm{V}\right\}}{\left\{\left(1000 \mathrm{pH}_{2 \mathrm{~S}} / \mathrm{RT}\right)+\left(\eta \mathrm{K}_{\mathrm{H} 2 \mathrm{~S}} \mathrm{pH}_{2 \mathrm{~S}} \mathrm{~V}\right)\right\}}\right] \times 150 \mathrm{day}^{-1}$

where $k=$ the second order rate constant in $\mathrm{L} \mathrm{mol}^{-1} \mathrm{~s}^{-1}$,

$\mathrm{p}_{\mathrm{H} 2 \mathrm{~S}}=$ the partial pressure of hydrogen sulfide in atm,

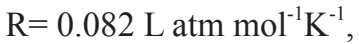

$\left[\mathrm{O}_{2}\right]=2.6 \times 10^{-4} \mathrm{~mol} \mathrm{~L}^{-1}$ at $25^{\circ} \mathrm{C}^{[73]}$ and

$2.4 \times 10^{-4} \mathrm{~mol} \mathrm{~L}^{-1}$ at $30^{\circ} \mathrm{C}^{[58]}$

$\eta=\left(1+\mathrm{K}_{1} /\left[\mathrm{H}^{+}\right]+\mathrm{K}_{1} \mathrm{~K}_{2} /\left[\mathrm{H}^{+}\right]^{2}\right)$,

$\mathrm{V}=10^{-3} \mathrm{~L}$ water per $\mathrm{m}^{3}$ of air,

$[\mathrm{S}]=$ the total concentration $\mathrm{H}_{2} \mathrm{~S}$ in solution $=\eta \mathrm{K}_{\mathrm{H} 2 \mathrm{~S}} \mathrm{p}_{\mathrm{H} 2 \mathrm{~S}}$,

$\mathrm{K}_{\mathrm{H} 2 \mathrm{~S}}=$ the Henry's law constant in $\mathrm{mol} \mathrm{L}^{-1} \mathrm{~atm}^{-1}$,

$\mathrm{K}_{1}$ and $\mathrm{K}_{2}$ are first and second dissociation constants of $\mathrm{H}_{2} \mathrm{~S}$.

The $\mathrm{K}_{\mathrm{H} 2 \mathrm{~S}}$ values are almost the same in the temperature range $25-30^{\circ} \mathrm{C}$ and, therefore, its value, $0.10132 \mathrm{~mol}$ $\mathrm{L}^{-1} \mathrm{~atm}^{-1}$, was used in all calculations at different temperatures ${ }^{[74]}$. At the reported ambient $\mathrm{p}_{\mathrm{H} 2 \mathrm{~S}}=30 \mathrm{ppb}$ ${ }^{[12]}$, the conversion rate were calculated at $\mathrm{pH}=6-11.45$ range and $\mathrm{T}=25-30^{\circ} \mathrm{C}$. The values (Table 7 ) show the conversion rates to be significant particularly when $\mathrm{pH}$ $\geq 8$ as depicted in Figure 7. It leads to an interesting conclusion that those areas where the rainwater $\mathrm{pH}$ is high the aquatic oxidation of $\mathrm{H}_{2} \mathrm{~S}$ in atmospheric aqueous system would be significant and $\mathrm{O}_{2}$ - oxidation pathway would be a significant contributor.

Our recent wet deposition studies found rainwater $\mathrm{pH}$ to be high in Western India ${ }^{[61,62]}$ and, therefore, the results of this study would be important for such regions globally. On the other hand, for the regions of low rainwater $\mathrm{pH}$ $<8.5$ such as North America this pathway would be insignificant ${ }^{[75]}$. 


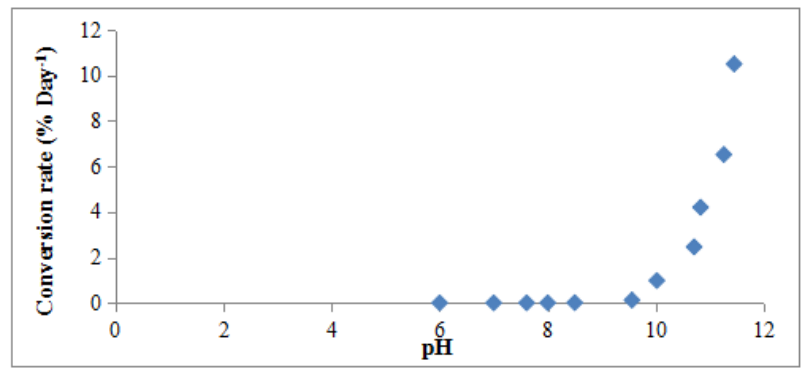

Figure 7. The plot of conversion rate of hydrogen sulfide versus $\mathrm{pH}$ at 25 and $30^{\circ} \mathrm{C}$

Table 7. The conversion rates $\left(\%\right.$ day $\left.^{-1}\right)$ of $\mathrm{H}_{2} \mathrm{~S}$ at 25 and $30^{\circ} \mathrm{C}$

\begin{tabular}{ccccc}
\hline $\mathrm{T},{ }^{\circ} \mathrm{C}$ & $\mathrm{pH}$ & $k\left(\mathrm{~L} \mathrm{~mol}^{-1} \mathrm{~s}^{-1}\right)$ & $\eta$ & $\%$ day $^{-1}$ \\
\hline 25 & 11.45 & $7.31 \times 10^{-2}$ & 28167 & 10.50 \\
25 & 10.83 & $11.8 \times 10^{-2}$ & 6570 & 4.20 \\
30 & 11.26 & $7.25 \times 10^{-2}$ & 17952 & 6.52 \\
30 & 10.71 & $9.60 \times 10^{-2}$ & 4974 & 2.46 \\
30 & 10.02 & $1.87 \times 10^{-1}$ & 1011 & 0.98 \\
30 & 9.56 & $6.53 \times 10^{-2}$ & 351 & 0.12 \\
30 & 8.50 & $4.59 \times 10^{-2}$ & 31 & $7.5 \times 10^{-3}$ \\
30 & 8.00 & $3.82 \times 10^{-2}$ & 10 & $2.1 \times 10^{-3}$ \\
30 & 7.61 & $4.16 \times 10^{-2}$ & 5 & $1.0 \times 10^{-3}$ \\
30 & 7.00 & $3.15 \times 10^{-2}$ & 1 & $1.5 \times 10^{-4}$ \\
30 & 6.00 & $1.04 \times 10^{-2}$ & 0.1 & $5.2 \times 10^{-6}$ \\
\hline
\end{tabular}

\section{Conclusions}

The major findings of this study are:

(1) The kinetics of oxidation of aqueous $\mathrm{H}_{2} \mathrm{~S}$ by $\mathrm{O}_{2}$ obeys the rate law:

$$
\frac{-\mathrm{d}\left[\mathrm{O}_{2}\right]}{\mathrm{dt}}=\frac{k_{1} \mathrm{~K}_{1}[\mathrm{H}+][\mathrm{S}]\left[\mathrm{O}_{2}\right]_{\mathrm{t}}}{\left(\left[\mathrm{H}_{+}\right]_{2}+\mathrm{K}_{1}[\mathrm{H}+]+\mathrm{K}_{1} \mathrm{~K}_{2}\right)}
$$

This is in conformity the reactive hydrogen sulfide species in aqueous medium being hydrogen sulfide ion, $\mathrm{HS}$.

(2) Our results indicate ammonium nitrate and anthropogenic VOCs such as acetanilide, benzene, ethanol, aniline, toluene, benzamide, $o$-xylene, $m$-xylene, $p$-xylene, anisole to have no significant effect.

(3) In this study, the involvement of trace metal catalysts is indicated by strong decrease in the rate of the reaction by EDTA, a strong chelating agent for metal ions.

(4) Both polar and non-polar mechanisms have been discussed.

(5) Calculation of atmospheric conversion rates shows these to increase with increase in $\mathrm{pH}$ and become sizable when $\mathrm{pH}$ is more than 6 .

\section{Acknowledgement}

This project SB/S4/AS:143/2014 was funded by Science and Engineering Research Board, Department of Science and Technology, Ministry of Science and Technology, Govt. of
India.

\section{References}

[1] World Health Organization. (2000). Hydrogen sulfide. In: Air quality guidelines, $2^{\text {nd }}$ Edition, WHO regional office for Europe, Copenhagen, Denmark, pp 146-147.

[2] Crutzen, P. J., \& Andreae, M. O. (1990). Biomass burning in the tropics: impact on atmospheric chemistry and biogeochemical cycles. Science, 250, 1669 1678. https://doi.org/10.1126/science.250.4988.1669.

[3] Gupta, K. S. (2012). Aqueous phase atmospheric oxidation of sulfur dioxide by oxygen: Role of trace atmospheric constituents - metals, volatile organic compounds and ammonia. Journal of Indian Chemical Society, 89, 713-724.

[4] Brandt, C., \& van Eldik R. (1995). Transition metal catalyzed oxidation of aqueous sulfur(IV) oxides. Atmospheric -relevant process and mechanisms. Chemical Reviews, 95, 119-190. https://doi.org/10.1021/ cr00033a006.

[5] Herrmann, H. (2003). Kinetics of aqueous phase reactions relevant to atmospheric chemistry. Chemical Reviews, 103, 4691-4716. https://doi.org/10.1021/ cr020658q.

[6] OECD. (1985). Chapter 1, Air, In: The state of the environment, organization for economic cooperation and development, Paris, pp 17-46.

[7] Wayne, R. P. (2000). Chemistry of atmospheres, Oxford University Press, London, pp 27, 366.

[8] Dhayal, Y., Chandel, C. P. S., \& Gupta, K. S. (2014). Role of some organic inhibitors on the oxidation of dissolved sulfur dioxide by oxygen in rain water medium. Environmental Science and Pollution Research, 21, 3474-3483. https://doi.org/10.1007/ s11356-013-2253-1.

[9] Baird, C., \& Cann, M. (2008). Environmental chemistry, W H Freeman, NewYork, pp 185-190.

[10] Bulfur, G., Boyle, A. J., \& Baldinger, L. H. (1936). The decomposition of solutions of sodium sulfide. The Journal of the American Pharmaceutical Association, 25, 1104-1106. https://doi.org/10.1002/ jps.3080251204.

[11] Weres, O., \& Tsao, L. (1983). Reaction of hydrogen sulfide with oxygen in the presence of sulfite. Earth science division, Lawrence Berkeley laboratory, University of California, pp 4-30. https://escholarship. org/content/qt71j1g6p8/qt71j1g6p8.pdf.

[12] ATSDR. (2006). Toxicological guide for hydrogen sulfide. Atlanta, GA, US Department of health and human services, agency for toxic substances and dis- 
ease registry.

[13] World Health Organization. (1981). Hydrogen sulfide, environmental health criteria, p. 19.

[14] Morse, J. W., Millero, F. J., Cornwell, J. C., \& Rickard, D. (1987). The chemistry of the hydrogen sulfide and iron sulfide system in natural waters. Earth Science Reviews, 24, 1-42. https://doi. org/10.1016/0012-8252(87)90046-8.

[15] Abel, E. (1956). Autoxidation in the sulfur group. Monatshefte Fur Chemie, 87, 498-502.

[16] Chen, K. Y., \& Morris, J. C. (1972). Kinetics of oxidation of aqueous sulfide by $\mathrm{O}_{2}$. Environmental Science and Technology, 6, 529-537. https://doi. org/10.1021/es60065a008.

[17] Avrahami, M., \& Golding, R. M. (1968). The oxidation of sulfide ion at very low concentrations in aqueous solutions. Journal of the Chemical Society A: Inorganic, Physical, Theoretical, 647-651. https://doi. org/10.1039/J19680000647.

[18] O’Brien, D. O., \& Birkner, F. B. (1977). Kinetics of oxygenation of reduced sulfur species in aqueous solution. Environmental Science and Technology, 11, 1114-1120. https://doi.org/10.1021/es60135a009.

[19] Alper, E., \& Ozturk, S. (1985). Kinetics of oxidation of aqueous sodium sulfide solutions by gaseous oxygen in a stirred cell reactor. Chemical Engineering Communications, 36, 343-349. https://doi. org/10.1080/00986448508911264.

[20] Wilmot, P. D., Cadee, K., Katinic, J. J., \& Kavanagh, B. V. (1988). Kinetics of sulfide oxidation by dissolved oxygen. Journal Water Pollution Control Federation, 60 1264-1270.

[21] Millero, F. J. (1986). The thermodynamics and kinetics of the hydrogen sulfide system in natural waters. Marine Chemistry, 18, 121-147. https://doi. org/10.1016/0304-4203(86)90003-4.

[22] Millero, F. J., Hubinger, S, Fernandez, M., \& Garnett, S. (1987). Oxidation of $\mathrm{H}_{2} \mathrm{~S}$ in seawater as a function of temperature, $\mathrm{pH}$ and ionic strength. Environmental Science and Technology, 21, 439-443. https://doi. org/10.1021/es00159a003.

[23] Millero, F. J. (1991). The oxidation of $\mathrm{H}_{2} \mathrm{~S}$ with $\mathrm{O}_{2}$ in the Black Sea. NATO ASI Series C: Mathematical and Physical Sciences. Black Sea Oceanography, 351, 205-227 https://link.springer.com/ book/10.1007/978-94-011-2608-3.

[24] Millero, F. J. (1991). The oxidation of hydrogen sulfide in Farmvaren Fjord. Limnology and Oceanography, 36, 1007-1014. https://doi.org/10.4319/ 10.1991.36.5.1007.

[25] Millero, F. J. (1991). The oxidation of hydrogen sulfide in the Chesapeake Bay. Estuarine, Coastal and Shelf Science, 33, 521-527. https://doi. org/10.1016/0272-7714(91)90088-S.

[26] Emerson, S., Cransto, R., \& Liss, P. S. (1979). Redox species in a reducing fjord: equilibrium and kinetic considerations. Deep-Sea Research, Part A: Oceanographic Research Papers, 26, 859-878.

[27] Leonov, A. V., \& Aizatullin, T. A. (1987). Kinetics and mechanism of hydrogen sulfide oxidation in sea water. Vodnye Resursy, 14, 89-103.

[28] Cline, J. D., \& Richards, F. A. (1969). Oxygenation of hydrogen sulfide in seawater of constant salinity, temperature and $\mathrm{pH}$. Environmental Science and Technology, 3, 838-843. https://doi.org/10.1021/ es60032a004.

[29] Ostlund, G. H., \& Alexander, J. (1963). Oxidation rate of sulfide in sea water preliminary study. Journal of Geophysical Research, 68, 3995-3997. https://doi. org/10.1029/JZ068i013p03995.

[30] Nielsen, A. H., Vollertsen, J., \& Hvitved-Jacobsen, T. (2006). Kinetics and stoichiometry of aerobic sulfide oxidation in wastewater from sewers - effects of $\mathrm{pH}$ and temperature. Water Environment Research, 78, 275-283. https://doi.org/10.2175/106143005x94367.

[31] Sharma, K. R., \& Yuan, Z. (2010). Kinetics of sulfide oxidation under high dissolved oxygen levels. Proceedings $6^{\text {th }}$ International conference on sewer processes and networks, Gold coast, Australia.

[32] Kim, C., Zhou, Q., Deng, B., Thornton, E. C., \& Xu, H. (2001) Chromium(VI) reduction by hydrogen sulfide in aqueous media: stoichiometry and kinetics. Environmental Science and Technology, 35, 22192225. https://doi.org/10.1021/es0017007.

[33] Sharma, V. K. (2010). Oxidation of inorganic compounds by ferrate(VI) and ferrate(V): one-electron and two-electron transfer steps. Environmental Science and Technology, 44, 5148-5152. https://doi. org/10.1021/es1005187.

[34] Cadena, F., \& Peters, R. W. (1988). Evaluation of chemical oxidizers for hydrogen sulfide control. Journal Water Pollution Control Federation, 60, 1259-1263.

[35] Betterton, E. A., \& Hoffmann, M. R. (1990). Kinetics and mechanism of the oxidation of aqueous hydrogen sulfide by peroxomonosulfate. Environmental Science and Technology, 24, 1819-1824. https://doi. org/10.1021/es00082a005.

[36] Tomar, M., \& Abdulah, T. H. A. (1994). Evaluation of chemicals to control the generation of malodorous hydrogen sulfide in waste water. Water Research, 28, 2545-2552. 
[37] Luther III, G. W., Findlay, A. J., Mcdonald, D. J., Owings, S. M., Hanson, T. E., Beinart, R. A., \& Girguis, P. R. (2011). Thermodynamics and kinetics of sulfide oxidation by oxygen: a look at inorganically controlled reactions and biologically mediated processes in the environment. Frontiers in Microbial Physiology and Metabolism, 2, 1-9. https://doi. org/10.3389/fmicb.2011.00062.

[38] Siang, H. Y., Tahir, N. M., Malek, A., \& Isa, M. Z. M. (2017). Breakdown of hydrogen sulfide in seawater under different ratio of dissolved oxygen / hydrogen sulfide. Malaysian Journal of Analytical Sciences, 21, 1016-1027. https://doi.org/10.17576/mjas-20172105-03.

[39] Nielsen, A. H., Vollertsen, J., \& Hvitved-Jacobsen, T. (2004). Chemical sulfide oxidation of wastewater - effects of $\mathrm{pH}$ and temperature. Water Science and Technology, 50, 185-192. https://pubmed.ncbi.nlm. nih.gov/15484760.

[40] Meena, V. K., Dhayal, Y., Rathore, D. S., Chandel, C. P. S., \& Gupta, K. S. (2017). Inhibition of aquated sulfur dioxide autoxidation by aliphatic, acyclic, aromatic, and heterocyclic volatile organic compounds. International Journal of Chemical Kinetics, 49, 221233. https://doi.org/10.1002/kin.21069.

[41] Meena, V. K., Dhayal, Y., Rathore, D. S., Chandel, C. P. S., \& Gupta, K. S. (2017). Inhibition of atmospheric aqueous phase autoxidation of sulphur dioxide by volatile organic compounds: mono-, di- and tri-substituted benzenes and benzoic acids. Progress in Reaction Kinetics and Mechanism, 42, 111-125. https:// doi.org/10.3184\%2F146867817X14806858832108.

[42] Meena, V. K., Dhayal, Y., Saxena, D., Rani, A., Chandel, C. P. S., \& Gupta, K. S. (2016). The influence of diesel - truck exhaust particles on the kinetics of the atmospheric oxidation of dissolved sulfur dioxide by oxygen. Environmental Science and Pollution Research, 23, 17380-17392. https://doi.org/10.1007/ s11356-016-6844-5.

[43] Grgic, I., Dovzan, A., Bercic, G., \& Hudnik, V. (1998). The effects of atmospheric organic compounds on the Fe-catalyzed S(IV) autoxidation in aqueous solution. Journal of Atmospheric Chemistry, 29, 315-337. https://link.springer.com/article/10.1023/A:1005918912994.

[44] Grgic, I., Podkrajsek, B., Barzaghi, P., \& Herrmann, H. (2007). Scavenging of $\mathrm{SO}_{4}{ }^{-}$radical anions by mono- and dicarboxylic acids in the Mn(II)-catalyzed S(IV) oxidation in aqueous solution. Atmospheric Environment, 41, 9187-9194. https://doi. org/10.1016/j.atmosenv.2007.07.051.
[45] Dhayal, Y., Chandel, C. P. S., \& Gupta, K. S. (2014). The influence of hydroxyl volatile organic compounds on the oxidation of aqueous sulfur dioxide by oxygen. Environmental Science and Pollution Research, 21, 7805-7817. https://doi.org/10.1007/ s11356-014-2661-x.

[46] Gupta, K. S., Mehta, R. K., Sharma, A. K., Mudgal, P. K., \& Bansal, S. P. (2008). Kinetics of uninhibited and ethanol-inhibited $\mathrm{CoO}, \mathrm{Co}_{2} \mathrm{O}_{3}$ and $\mathrm{Ni}_{2} \mathrm{O}_{3}$ catalyzed autoxidation of sulfur(IV) in alkaline medium. Transition Metal Chemistry, 33, 809-817. https://doi. org/10.1007/s10874-009-9123-8.

[47] Martin, L. R., Hill, M. W., Tai, A. F., \& Good, T. W. (1991). The iron catalyzed oxidation of sulfur(IV) in aqueous solution: Differing effects of organics at high and low pH. Journal of Geophysical Resarch, 96, 3085-3097. https://doi.org/10.1029/90JD02611.

[48] Pasiuk- Bronikowska, W., Bronikowska, T., \& Ulejczyk, M. (2003). Synergy in the autoxdation of S(IV) inhibited by phenolic compounds. Journal of Physical Chemistry A, 107, 1742-1748. https://doi. org/10.1021/jp0208790.

[49] Pasiuk- Bronikowska, W., Bronikowska, T., \& Ulejczyk, M. (2003). Inhibition of the S(IV) autoxidation in the atmosphere by secondary terpeinic compounds. Journal of Atmospheric Chemistry, 44, 97-111. https://link.springer.com/article/10.1023/ A:1022164702310.

[50] Podkrajsek, B., Grgic, I., Tursic, J., \& Bercic, G. (2006). Influence of atmospheric carboxylic acids on catalytic oxidation of sulfur(IV). Journal of Atmospheric Chemistry, 54, 103-120. https://doi.org 10.1007/s10874-006-9018-x.

[51] Rudizinski, K. J. (2004). Degradation of isoprene in the presence of sulphoxy radical anions. Journal of Atmospheric Chemistry, 48, 191-216. https://doi. org/10.1023/B:JOCH.0000036851.98523.ef.

[52] Wang, L.-D., Yi, Z., Ma, Y.-L., \& Ji-Ming, H. (2008). Intrinsic kinetics of sulfite oxidation with inhibitor of phenol. Acta Chimica Sinica, 66, 2336-2340.

[53] Wang, L.-D., Ma, Y.-L., Ji-Ming, H., \& Yi, Z. (2009). Mechanism and kinetics of sulfite oxidation in the presence of ethanol. Industrial and Engineering Chemistry Research, 48, 4307-4311. https://doi. org/10.1021/ie801731h.

[54] Wolf, A., Deutsch, F., Hoffmann, P., \& Ortner, H. M. (2000). The influence of oxalate on Fe-catalyzed $\mathrm{S}(\mathrm{IV})$ oxidation by oxygen in aqueous solution. Journal of Atmospheric Chemistry, 37, 125-135. https:// doi.org/10.1023/A:1006462025384.

[55] Ziajka, J., Beer, F., \& Warneck, P. (1994). Iron-cat- 
alyzed oxidation of bisulfate aqueous solution: evidence for a free radical chain mechanism. Atmospheric Environment, 28, 2549-2552. https://doi. org/10.1016/1352-2310(94)90405-7.

[56] Ziajka, J., \& Pasiuk-Bronikowska, W. (2005). Rate constants for atmospheric trace organics scavenging $\mathrm{SO}_{4}{ }^{-}$in the Fe-catalyzed autoxidation of S(IV). Atmospheric Environment, 39, 1431-1438. https://doi. org/10.1016/j.atmosenv.2004.11.024.

[57] Ziajka, P., \& Pasiuk-Bronikowska, W. (2003). Autoxidation of sulfur dioxide in the presence of alcohols under conditions related to tropospheric aqueous phase. Atmospheric Environment, 37, 3913-3922. https://doi.org/10.1016/S1352-2310(03)00503-X.

[58] Mudgal, P. K., Bansal, S. P., \& Gupta, K. S. (2007). Kinetics of atmospheric oxidation of nitrous acid by oxygen in aqueous medium. Atmospheric Environment, 41, 4097-4105. https://doi.org/10.1016/j.atmosenv.2007.01.036.

[59] Hoffmann, M. R. (1980). Trace metal catalysis in aquatic environments. Environmental Science and Technology, 14, 1061-1066. https://doi.org/10.1021/ es60169a007.

[60] Zhang, J. Z., \& Millero, F. J. (1993). Kinetics of oxidation of hydrogen sulfide in natural waters. In: Alpers, C.N., \& Blowes, D.W. (Eds) Environmental Geochemisry of Sulfide Oxidation, Chapter 26, American Chemical Society, Washington, DC pp 393-409.

[61] Manoj, S. V., Mishra, C. D., Sharma, M., Rani, A., Jain, R., Bansal, S. P., \& Gupta, K. S. (2000). Iron, manganese and copper concentrations in wet precipitations and kinetics of the oxidation of $\mathrm{SO}_{2}$ in rain water at two urban sites, Jaipur and Kota, in western India. Atmospheric Environment, 34, 4479-4486. https://doi.org/10.1016/S1352-2310(00)00117-5.

[62] Misra, C. D., Khanooja, P. S., Sharma, A. K., Mudgal, P. K., Bansal, S. P., \& Gupta, K. S. (2013). Studies on rainwater and dry depositions at Jaipur, India. Journal of Indian Chemical Society, 90, 1137-1146. https://www.researchgate.net/publication/287410793.

[63] Vazquez, G., Zhang, J., \& Millaro, F. J. (1989). Effect of metals on the rate of the oxidation of $\mathrm{H}_{2} \mathrm{~S}$ in seawater. Geophysical Research Letters, 16, 13631366. https://doi.org/10.1029/GL016i012p01363.

[64] Mudgal, P. K., Sharma, A. K., Mishra, C. D., Bansal, S. P., \& Gupta, K. S. (2008). Kinetics of ammonia and ammonium ion inhibition of the atmospheric oxidation of aqueous sulfur dioxide by oxygen. Journal of Atmospheric Chemistry, 61, 31-55. https://doi. org/10.1007/s10874-009-9123-8.

[65] Chen, K. Y., \& Morris, J. C. (1972). Oxidation of sulfide by $\mathrm{O}_{2}$ : catalysis and inhibition. Journal of the Sanitary Engineering Division, 98, 215-227.

[66] Sun, W., Nesic, S., Young, D., \& Woollam, R. C. (2008). Equilibrium expressions related to the solubility of the sour corrosion product mackinawite. Industrial and Engineering Chemistry Research, 47, 1738-1742. https://doi.org/10.1021/ie070750i.

[67] Harrup, M. K., \& Hill, C. L. (1994). Polyoxometalate Catalysis of the Aerobic Oxidation of Hydrogen Sulfide to Sulfur. Inorganic Chemistry, 33, 5448-5455. https://doi.org/10.1021/ie070750i.

[68] Lewis, A. E. (2010). Review of metal sulphide precipitation. Hydrometallurgy, 104, 222-234. https:// doi.org/10.1016/j.hydromet.2010.06.010.

[69] Steijns, M., Koopman, P., Nieuwenhuijse, B., \& Mars, P. (1976). The mechanism of the catalytic oxidation of hydrogen sulfide III. An electron spin resonance study of the sulfur catalyzed oxidation of hydrogen sulfide. Journal of Catalysis, 42, 96-106. https://doi.org/10.1016/0021-9517(76)90095-6.

[70] Hoffmann, M. R., \& Lim B. C. (1979). Kinetics and mechanism of the oxidation of sulfide by oxygen: catalysis by homogenous metal-phthalocyanine complexes. Environmental Science and Technology, 13, 1406-1414. https://doi.org/10.1021/es60159a014.

[71] Bielski, B. H. J., Cabelli, D. E., Arudi, R. L., \& Ross A. B. (1985). Reactivity of $\mathrm{HO}_{2} / \mathrm{O}_{2}^{-}$radicals in aqueous solutions. Journal of Physical and Chemical Reference Data, 14, 1041-1100. https://doi. org/10.1063/1.555739.

[72] Hoffmann, M. R., \& Hong, A. P. K. (1987). Catalytic oxidation of reduced sulfur compounds by homogeneous and heterogeneous $\mathrm{Co}$ (II) phthalocyanine complexes. Science of the Total Environment, 64, 99115. https://doi.org/10.1016/0048-9697(87)90125-2.

[73] Patel, H., \& Vashi, R. T. (2015). Characterization of textile wastewater. In: Characterization and treatment of textile wastewater. Butterworth Heinemann, Waltham, pp 21-71.

[74] Sander, R. (2015). Compilation of Henry's law constants (version 4.0) for water as solvent. Atmospheric Chemistry \& Physics, 15, 4399-4981. https://doi. org/10.5194/acp-15-4399-2015.

[75] Jacob, D. J. (1999). Acid rain. In: Introduction to atmospheric chemistry. Princeton University Press, New Jersey, pp 247-255. 